Proceedings of the Edinburgh Mathematical Society (2003) 46, 117-146 (C)

DOI:10.1017/S0013091501000426 Printed in the United Kingdom

\title{
THE BEST-CONSTANT PROBLEM FOR A FAMILY OF GAGLIARDO-NIRENBERG INEQUALITIES ON A COMPACT RIEMANNIAN MANIFOLD
}

\author{
CHRISTOPHE BROUTTELANDE \\ Université Paul Sabatier, Département de Mathématiques, 118 route de Narbonne, \\ 31062 Toulouse Cedex 4, France (Christophe.Brouttelande@math.ups-tlse.fr)
}

(Received 5 April 2001)

\begin{abstract}
The best-constant problem for Nash and Sobolev inequalities on Riemannian manifolds has been intensively studied in the last few decades, especially in the compact case. We treat this problem here for a more general family of Gagliardo-Nirenberg inequalities including the Nash inequality and the limiting case of a particular logarithmic Sobolev inequality. From the latter, we deduce a sharp heat-kernel upper bound.
\end{abstract}

Keywords: Sobolev logarithmic inequality; Gagliardo-Nirenberg inequalities;

best-constant problem; optimal inequalities

AMS 2000 Mathematics subject classification: Primary 58J05

\section{Introduction}

\subsection{The case of the Euclidean space $\mathbb{R}^{n}$}

Let $p$ be a positive real number. If $n>p$, the $H_{1}^{p}\left(\mathbb{R}^{n}\right)$ Sobolev inequality asserts that there exists a constant $A$ such that for all $u \in H_{1}^{p}\left(\mathbb{R}^{n}\right)$,

$$
\left(\int_{\mathbb{R}^{n}}|u|^{n p /(n-p)} \mathrm{d} x\right)^{(n-p) / n p} \leqslant A\left(\int_{\mathbb{R}^{n}}|\nabla u|^{p} \mathrm{~d} x\right)^{1 / p} .
$$

When combining with Hölder's inequality, we obtain a new family of inequalities, called Gagliardo-Nirenberg inequalities, asserting that for all $u \in H_{1}^{p}\left(\mathbb{R}^{n}\right)$,

$$
\left(\int_{\mathbb{R}^{n}}|u|^{r} \mathrm{~d} x\right)^{1 / r} \leqslant\left(A \int_{\mathbb{R}^{n}}|\nabla u|^{p} \mathrm{~d} x\right)^{\theta / 2}\left(\int_{\mathbb{R}^{n}}|u|^{s} \mathrm{~d} x\right)^{(1-\theta) / s},
$$

where $r, s>0, \theta \in[0,1]$ and

$$
\frac{1}{r}=\frac{\theta}{q}+\frac{1-\theta}{s} .
$$

Actually, according to [3], when $p$ is fixed and $\theta>0$, these inequalities are all equivalent up to the constant $A$. Some famous particular cases have numerous applications. One 
may mention Nash's inequality,

$$
\left(\int_{\mathbb{R}^{n}}|u|^{2} \mathrm{~d} x\right)^{1+(2 / n)} \leqslant A\left(\int_{\mathbb{R}^{n}}|\nabla u|^{2} \mathrm{~d} x\right)\left(\int_{\mathbb{R}^{n}}|u| \mathrm{d} x\right)^{4 / n},
$$

introduced by Nash in his celebrated paper [13], which is obtained by setting $r=2$, $s=1$ and $\theta=n /(n+2)$. If $r=2+(4 / n), s=2$ and $\theta=n /(n+2)$, we then obtain the inequality

$$
\int_{\mathbb{R}^{n}}|u|^{2+(4 / n)} \mathrm{d} x \leqslant A\left(\int_{\mathbb{R}^{n}}|\nabla u|^{2} \mathrm{~d} x\right)\left(\int_{\mathbb{R}^{n}}|u|^{2} \mathrm{~d} x\right)^{2 / n}
$$

which has been used by Moser in a subsequent work [12]. Let us note that these inequalities still hold when $n \leqslant p$ (which implies $\theta \neq 1$ ), whereas the Sobolev embeddings are not valid in this case. One can refer to [3], for example, for a more general discussion. In the following, we restrict $p$ to $p=2$ and thus consider, when $\theta \neq 0$, the inequality

$$
\left(\int_{\mathbb{R}^{n}}|u|^{r} \mathrm{~d} x\right)^{2 / r \theta} \leqslant A\left(\int_{\mathbb{R}^{n}}|\nabla u|^{2} \mathrm{~d} x\right)\left(\int_{\mathbb{R}^{n}}|u|^{s} \mathrm{~d} x\right)^{2(1-\theta) / s \theta} .
$$

Let us fix $r$ and assume that (1.1) holds with an $A$ independent of $\theta$, which is the case for all $n>0$ (see [3]). Making $\theta$ go to 0 , we obtain that for all $u>0$ such that $\|u\|_{r}=1$ the logarithmic Sobolev inequality

$$
\int_{\mathbb{R}^{n}} u^{r} \ln u^{r} \mathrm{~d} x \leqslant\left(\frac{2}{n}+\frac{2-r}{r}\right)^{-1} \ln \left(A \int_{\mathbb{R}^{n}}|\nabla u|^{2} \mathrm{~d} x\right) .
$$

According to [3], this inequality is again equivalent to the previous ones and we shall thus consider that it represents the case $\theta=0$.

Let $A_{0}(r, s, \theta, n)$ be the optimum $A$ such that (1.1) is valid. In most cases its explicit value is unknown. The best constant in Sobolev inequalities was first obtained independently by Aubin $[\mathbf{1}]$ and by Talenti $[\mathbf{1 4}]$ when $n \geqslant 3$. They showed that

$$
A_{0}\left(\frac{2 n}{n-2}, s, 1, n\right)=K(n, 2)^{2}=\frac{4}{n(n-2) \omega_{n}^{2 / n}},
$$

where $\omega_{n}$ is the volume of the standard unit sphere of dimension $n$. Later, the $S L_{2, n}$ case was solved by Carlen [4]. In addition, with Loss [5] he computed the best constant for Nash's inequality. These values are

$$
\begin{aligned}
A_{0}(2,2,0, n) & =\frac{2}{n \pi \mathrm{e}} \\
A_{0}\left(2,1, \frac{n}{n+2}, n\right) & =\frac{(n+2)^{(n+2) / n}}{2^{2 / n} n \lambda_{1}(\mathcal{B})|\mathcal{B}|^{2 / n}},
\end{aligned}
$$

where $\lambda_{1}(\mathcal{B})$ is the first Neumann eigenvalue of the Laplacian for radial functions on the unit ball $\mathcal{B}$ in $\mathbb{R}^{n}$ and $|\mathcal{B}|$ is the volume of $\mathcal{B}$ in $\mathbb{R}^{n}$. One may remark that $\lambda_{1}(\mathcal{B})$ can be numerically computed. A brief discussion about this last point can be found in [5]. 


\subsection{The Riemannian case}

Let $(M, g)$ be a smooth compact Riemannian $n$-manifold. When $n \geqslant 3$, the $H_{1}^{2}$ Sobolev inequality on $M$ asserts that there exist constants $A$ and $B$ such that for all $u \in H_{1}^{2}(M)$,

$$
\left(\int_{M}|u|^{2 n /(n-2)} \mathrm{d} v_{g}\right)^{(n-2) / n} \leqslant A \int_{M}|\nabla u|_{g}^{2} \mathrm{~d} v_{g}+B \int_{M}|u|^{2} \mathrm{~d} v_{g} .
$$

As in the case of the Euclidean space $\mathbb{R}^{n}$, we can define all the Gagliardo-Nirenberg inequalities on $M$ by Hölder's inequality. Actually, we obtain that for all $u \in H_{1}^{2}(M)$,

$$
\left(\int_{M}|u|^{r} \mathrm{~d} v_{g}\right)^{2 / r \theta} \leqslant\left(A \int_{M}|\nabla u|_{g}^{2} \mathrm{~d} v_{g}+B \int_{M}|u|^{2} \mathrm{~d} v_{g}\right)\left(\int_{M}|u|^{s} \mathrm{~d} v_{g}\right)^{2(1-\theta) / s \theta}
$$

where $r, s>0, \theta \in(0,1)$ and

$$
\frac{1}{r}=\frac{\theta(n-2)}{2 n}+\frac{1-\theta}{s}
$$

Again, these inequalities are all equivalent and can be defined for all $n \geqslant 1$. For the last assertion, one should refer to Theorem 1.1 in [8] (which treats the case of a modified Nash inequality) for an easy-to-adapt proof using a partition-of-unity argument.

Now, we define

$$
\mathcal{A}(r, s, \theta, n)=\{A \in \mathbb{R} \text { s.t. } \exists B \in \mathbb{R} \text { for which (1.3) is valid }\} .
$$

One may ask if this set is closed and what is its infimum, called the first best constant. This problem has been intensively studied for the Sobolev inequalities (a complete discussion may be found in [10]). Recently, Humbert [11] solved the Nash case. In both cases, it was shown that the set is closed and that the infimum is the best constant of the corresponding Euclidean inequalities. In these proofs, the explicit value of the best constant was known but not used. Therefore, one we may ask if the answer is identical for all the Gagliardo-Nirenberg inequalities. The first aim of this paper is to study to what extent the previous proofs may be generalized to other cases. At the same time, we point out the fact that the explicit value of $A_{0}(r, s, \theta, n)$ is useless for solving the first best-constant problem for the family of inequalities that we study.

One may easily check that $\inf \mathcal{A}(r, s, \theta, n)=A_{0}(r, s, \theta, n)$. To this end, we may again simply follow the proof of Theorem 1.1 in [8]. Our main result in this work is to give conditions on $r, s, \theta$ such that (1.3) holds with $A=A_{0}(r, s, \theta, n)$, including the Nash case studied by Humbert [11]. The proof we present does not allow us to treat the full range of parameters. It generalizes [11], itself inspired by the paper by Druet [7]. While the main ideas of the proof below are already present in these works, the range of parameters $r, s, \theta$ under investigation presents us with a number of new technical difficulties. For the sake of completeness, we thus decided to present a self-contained proof. Our main result is the following. 
Theorem 1.1. Let $(M, g)$ be a smooth compact Riemannian n-manifold. Let $r, s, \theta$ be constants satisfying $r \geqslant 2, s \geqslant 1, \theta \in(0,1)$ and

$$
\frac{1}{r}=\frac{\theta(n-2)}{2 n}+\frac{1-\theta}{s} .
$$

If $s \leqslant 2 \leqslant r<2+s(2 / n)$, then there exists a constant $B$ such that for all $u \in C^{\infty}(M)$,

$$
\left(\int_{M}|u|^{r} \mathrm{~d} v_{g}\right)^{2 / r \theta} \leqslant\left(A_{0}(r, s, \theta, n) \int_{M}|\nabla u|_{g}^{2} \mathrm{~d} v_{g}+B \int_{M}|u|^{2} \mathrm{~d} v_{g}\right)\left(\int_{M}|u|^{s} \mathrm{~d} v_{g}\right)^{2(1-\theta) / s \theta} .
$$

Let us now study some interesting particular cases. The Nash inequality is obviously included in our family but we can remark that Moser's inequality only appears as a limiting case. Indeed, we then have $r=2+s(2 / n)$. Up to now, we have not been able to prove that $B$ does not explode as $A$ goes to $A_{0}(r, s, \theta, n)$. Another limiting case can be treated with this theorem: the logarithmic Sobolev inequality. This one is obtained as in $\S 1.1$, by fixing $r=2$ and making $\theta$ go to 0 . The following result will be proved in $\S 3$.

Corollary 1.2. Let $(M, g)$ be a smooth compact Riemannian n-manifold. There exists a constant $B$ such that for all $u \in C^{\infty}(M)$ verifying $u>0$ and $\|u\|_{2}=1$,

$$
\int_{M} u^{2} \ln u^{2} \mathrm{~d} v_{g} \leqslant \frac{1}{2} n \ln \left(\frac{2}{n \pi \mathrm{e}} \int_{M}|\nabla u|_{g}^{2} \mathrm{~d} v_{g}+B\right) .
$$

The best-constant problem for the Sobolev inequality has as many applications as the Yamabe problem. A classical use of the logarithmic Sobolev inequalities is the computation of heat-kernel upper bounds (see, for example, $[\mathbf{2 , 6}]$ ). Actually, following a result of Bakry [2], the optimal Euclidean inequality can be used to compute the optimal upper bound

$$
\left\|P_{t}\right\|_{1, \infty} \leqslant \frac{1}{(4 \pi t)^{n / 2}}
$$

where $\left(P_{t}\right)_{t>0}$ is the heat semigroup on the Euclidean space $\mathbb{R}^{n}$. One may ask if a similar argument works on manifolds. At first, in $\S 3.2$, we shall cite the theorem obtained by Bakry [2]. From it and Corollary 1.2, we will then deduce the following.

Corollary 1.3. Let $(M, g)$ be a smooth compact Riemannian $n$-manifold and let $\left(P_{t}\right)_{t>0}$ be the heat semigroup on $M$. One then has

$$
\left\|P_{t}\right\|_{1, \infty} \leqslant \frac{1}{(4 \pi t)^{n / 2}} \mathrm{e}^{\left(n \pi \mathrm{e} B_{0} / 3\right) t},
$$

where $0<t \leqslant\left(\pi \mathrm{e} B_{0}\right)^{-1}$ and $B_{0}$ is the best constant $B$ in (1.4). 


\section{Proof of Theorem 1.1}

As already indicated, the proof follows the pattern of the proof of the main result of [11], itself inspired by [7]. As $r, s, \theta$ and $n$ are fixed in this section, we shall denote by $A_{0}$ the constant $A_{0}(r, s, \theta, n)$. The case $n=1$ is handled with a partition-of-unity argument as we prove that $A_{0}$ is the infimum of $A(r, s, \theta, n)$. One can thus assume that $n \geqslant 2$. Without loss of generality, we can also assume that $\operatorname{Vol}_{g}(M)=1$. Moreover, let us observe that $\theta \in(0,1)$ implies $s<r$. We proceed here by contradiction. The proof is composed of three steps. The first one is a preliminary step in which we introduce alternative notation that will be used throughout this section. This part being nearly identical to the one in [11], we keep the notation from that paper to make comprehension easier. Step 2 is a set of nine lemmas. The first three are classical ones and deal with concentration-point phenomena in partial differential equations, whereas the other six give more specific results. We then conclude in the third step.

Step 1. Preliminary.

Proceeding by contradiction, we assume that for all $B>0$ there exists $u \in C^{\infty}(M)$ such that

$$
\left(\int_{M}|u|^{r} \mathrm{~d} v_{g}\right)^{2 / r \theta}>\left(A_{0} \int_{M}|\nabla u|_{g}^{2} \mathrm{~d} v_{g}+B \int_{M}|u|^{2} \mathrm{~d} v_{g}\right)\left(\int_{M}|u|^{s} \mathrm{~d} v_{g}\right)^{2(1-\theta) / s \theta} .
$$

This is equivalent to

$$
\mu_{\alpha}=\inf _{u \in \mathcal{H}} I_{\alpha}<A_{0}^{-1}
$$

for all $\alpha>0$, where

and

$$
I_{\alpha}=\left(\int_{M}|\nabla u|_{g}^{2} \mathrm{~d} v_{g}+\alpha \int_{M}|u|^{2} \mathrm{~d} v_{g}\right)\left(\int_{M}|u|^{s} \mathrm{~d} v_{g}\right)^{2(1-\theta) / s \theta}
$$

$$
\mathcal{H}=\left\{u \in C^{\infty}(M) / \int_{M}|u|^{r} \mathrm{~d} v_{g}=1\right\} .
$$

We assume throughout the proof that $s>1$, the case $s=1$ being handled by replacing $s$ with $1+\epsilon_{\alpha}$ in $I_{\alpha}$, where $\left(\epsilon_{\alpha}\right)_{\alpha}$ is such that $\lim \epsilon_{\alpha}=0$ (see [11] for the particular case $r=2$ and $s=1)$. Using the same arguments as in [8], we can prove that there exists $u_{\alpha} \in H_{1}^{2}(M), u_{\alpha}>0$, such that $I_{\alpha}\left(u_{\alpha}\right)=\mu_{\alpha}$. Moreover, in the sense of distributions,

$$
2 A_{\alpha} \Delta_{g} u_{\alpha}+2 \alpha A_{\alpha} u_{\alpha}+\frac{2(1-\theta)}{\theta} B_{\alpha} u_{\alpha}^{s-1}=k_{\alpha} u_{\alpha}^{r-1},
$$

where

$$
\begin{aligned}
A_{\alpha} & =\left(\int_{M} u_{\alpha}^{s} \mathrm{~d} v_{g}\right)^{2(1-\theta) / s \theta} \\
B_{\alpha} & =\left(\int_{M}\left|\nabla u_{\alpha}\right|_{g}^{2} \mathrm{~d} v_{g}+\alpha \int_{M} u_{\alpha}^{2} \mathrm{~d} v_{g}\right)\left(\int_{M} u_{\alpha}^{s} \mathrm{~d} v_{g}\right)^{(2(1-\theta) / s \theta)-1}, \\
k_{\alpha} & =\left(\frac{2}{\theta}\right) \mu_{\alpha} .
\end{aligned}
$$


The Sobolev embedding theorems and the standard elliptic theory (see [9]) imply $u_{\alpha} \in$ $C^{2}(M)$. From now on, all limits below are taken as $\alpha \rightarrow \infty$. Considering subsequences if needed, we can assume that all sequences have limits (finite or infinite).

One has $\mu_{\alpha}<A_{0}^{-1}$, hence

$$
\lim \left(\int_{M} u_{\alpha}^{2} \mathrm{~d} v_{g}\right)\left(\int_{M} u_{\alpha}^{s} \mathrm{~d} v_{g}\right)^{2(1-\theta) / s \theta}=0
$$

and

$$
\limsup \left(\int_{M}\left|\nabla u_{\alpha}\right|_{g}^{2} \mathrm{~d} v_{g}\right)\left(\int_{M} u_{\alpha}^{s} \mathrm{~d} v_{g}\right)^{2(1-\theta) / s \theta} \leqslant A_{0}^{-1} .
$$

From (1.3) with $A=A_{0}+\epsilon, B=B_{\epsilon}$ and $u=u_{\alpha}$ with $\epsilon$ small, we obtain

$$
\left(A_{0}+\epsilon\right)^{-1} \leqslant\left(\int_{M}\left|\nabla u_{\alpha}\right|_{g}^{2} \mathrm{~d} v_{g}+\frac{B_{\epsilon}}{A_{0}+\epsilon} \int_{M}\left|u_{\alpha}\right|^{2} \mathrm{~d} v_{g}\right)\left(\int_{M}\left|u_{\alpha}\right|^{s} \mathrm{~d} v_{g}\right)^{2(1-\theta) / s \theta}
$$

Hence

$$
\liminf \left(\int_{M}\left|\nabla u_{\alpha}\right|_{g}^{2} \mathrm{~d} v_{g}\right)\left(\int_{M} u_{\alpha}^{s} \mathrm{~d} v_{g}\right)^{2(1-\theta) / s \theta} \geqslant A_{0}^{-1}
$$

As a consequence,

$$
\begin{aligned}
& \lim A_{\alpha} \int_{M}\left|\nabla u_{\alpha}\right|_{g}^{2} \mathrm{~d} v_{g}=A_{0}^{-1}, \\
& \lim B_{\alpha} \int_{M} u_{\alpha}^{s} \mathrm{~d} v_{g}=\lim B_{\alpha} A_{\alpha}^{s \theta / 2(1-\theta)}=A_{0}^{-1}, \\
& \lim k_{\alpha}=(2 / \theta) A_{0}^{-1}, \\
& \lim \alpha A_{\alpha} \int_{M} u_{\alpha}^{2} \mathrm{~d} v_{g}=0 .
\end{aligned}
$$

Let $x_{\alpha} \in M$ be such that $u_{\alpha}\left(x_{\alpha}\right)=\left\|u_{\alpha}\right\|_{\infty}$. Set $a_{\alpha}=\left(A_{\alpha}\left\|u_{\alpha}\right\|_{\infty}^{2-r}\right)^{1 / 2}$. Since

$$
1=\int_{M} u_{\alpha}^{r} \mathrm{~d} v_{g} \leqslant \int_{M} u_{\alpha}^{2} \mathrm{~d} v_{g}\left\|u_{\alpha}\right\|_{\infty}^{r-2}
$$

we obtain from (2.5) that $a_{\alpha} \rightarrow 0$.

Step 2. Some lemmas.

The first three results are classical. One begins with the following.

Lemma 2.1. For all $\delta>0$,

$$
\lim \frac{\int_{B_{x_{\alpha}}\left(\delta a_{\alpha}\right)} u_{\alpha}^{s} \mathrm{~d} v_{g}}{\int_{M} u_{\alpha}^{s} \mathrm{~d} v_{g}}>0 .
$$


Proof. Let $\delta>0$. For all $x \in B(0, \delta)$, set

$$
\begin{aligned}
& g_{\alpha}(x)=\left(\exp _{x_{\alpha}}^{*} g\right)\left(a_{\alpha} x\right), \\
& \varphi_{\alpha}(x)=\left\|u_{\alpha}\right\|_{\infty}^{-1} u_{\alpha}\left(\exp _{x_{\alpha}}\left(a_{\alpha} x\right)\right) .
\end{aligned}
$$

It is an easy matter to check that

$$
\begin{aligned}
\Delta_{g_{\alpha}} \varphi_{\alpha}(x)=\left\|u_{\alpha}\right\|_{\infty}^{-1} a_{\alpha}^{2} \Delta_{g} u_{\alpha}\left(\exp _{x_{\alpha}}\left(a_{\alpha} x\right)\right) \\
=\left\|u_{\alpha}\right\|_{\infty}^{1-r}\left(\frac{1}{2} k_{\alpha} u_{\alpha}\left(\exp _{x_{\alpha}}\left(a_{\alpha} x\right)\right)^{r-1}\right. \\
\left.\quad \quad-\frac{1-\theta}{\theta} B_{\alpha} u_{\alpha}\left(\exp _{x_{\alpha}}\left(a_{\alpha} x\right)\right)^{s-1}-\alpha A_{\alpha} u_{\alpha}\left(\exp _{x_{\alpha}}\left(a_{\alpha} x\right)\right)\right) .
\end{aligned}
$$

Hence

$$
\Delta_{g_{\alpha}} \varphi_{\alpha}+\alpha A_{\alpha} \varphi_{\alpha}\left\|u_{\alpha}\right\|_{\infty}^{2-r}+\frac{1-\theta}{\theta}\left\|u_{\alpha}\right\|_{\infty}^{s-r} B_{\alpha} \varphi_{\alpha}^{s-1}=\frac{1}{2} k_{\alpha} \varphi_{\alpha}^{r-1} .
$$

Noting that $\Delta_{g} u_{\alpha}\left(x_{\alpha}\right) \geqslant 0$, we obtain from (2.1) that

$$
\alpha A_{\alpha}+\frac{1-\theta}{\theta} B_{\alpha}\left\|u_{\alpha}\right\|_{\infty}^{s-2} \leqslant \frac{1}{2} k_{\alpha}\left\|u_{\alpha}\right\|_{\infty}^{r-2},
$$

which implies that $\left|\Delta_{g_{\alpha}} \varphi_{\alpha}\right| \leqslant C$. By standard elliptic arguments (see, for example, [9]), we then show that the sequence $\left(\varphi_{\alpha}\right)$ is equicontinuous. Hence, by the Ascoli theorem, there exists $\varphi \in C^{0}(B(0, \delta))$ such that $\varphi_{\alpha} \rightarrow \varphi$ in $C^{0}(B(0, \delta))$. Moreover,

$$
\varphi(0)=\lim \varphi_{\alpha}(0)=1 \text {. }
$$

Therefore,

$$
\begin{aligned}
\int_{B(0, \delta)} \varphi_{\alpha}^{s} \mathrm{~d} v_{g_{\alpha}} & =\left\|u_{\alpha}\right\|_{\infty}^{-s} a_{\alpha}^{-n} \int_{B_{x_{\alpha}}\left(a_{\alpha} \delta\right)} u_{\alpha}^{s} \mathrm{~d} v_{g} \\
& =\left\|u_{\alpha}\right\|_{\infty}^{-s-(2-r)(n / 2)} A_{\alpha}^{-(n / 2)+(s \theta / 2(1-\theta))} \frac{\int_{B_{x_{\alpha}}\left(a_{\alpha} \delta\right)} u_{\alpha}^{s} \mathrm{~d} v_{g}}{\int_{M} u_{\alpha}^{s} \mathrm{~d} v_{g}} .
\end{aligned}
$$

Using the relations

$$
\begin{gathered}
\frac{2}{n}=1-\frac{2}{r \theta}+\frac{2(1-\theta)}{s \theta} \\
(r-s) \frac{1}{2} n \frac{2(1-\theta)}{s \theta}-(2-r) \frac{1}{2} n=r
\end{gathered}
$$

we obtain

$$
\int_{B(0, \delta)} \varphi_{\alpha}^{s} \mathrm{~d} v_{g_{\alpha}}=\left(\left\|u_{\alpha}\right\|_{\infty}^{r-s} A_{\alpha}^{s \theta / 2(1-\theta)}\right)^{1-(n(1-\theta) / s \theta)} \frac{\int_{B_{x_{\alpha}}\left(a_{\alpha} \delta\right)} u_{\alpha}^{s} \mathrm{~d} v_{g}}{\int_{M} u_{\alpha}^{s} \mathrm{~d} v_{g}} .
$$

One may easily verify that

$$
r<2+s \frac{2}{n} \Leftrightarrow \frac{2}{r \theta}>1 \quad \Leftrightarrow \quad 1-\frac{n(1-\theta)}{s \theta}<0 .
$$


Since (2.3) and (2.6) imply that $A_{\alpha}^{-s \theta / 2(1-\theta)} \leqslant C\left\|u_{\alpha}\right\|_{\infty}^{r-s}$, we have

$$
\int_{B(0, \delta)} \varphi_{\alpha}^{s} \mathrm{~d} v_{g_{\alpha}} \leqslant C \frac{\int_{B_{x_{\alpha}}\left(a_{\alpha} \delta\right)} u_{\alpha}^{s} \mathrm{~d} v_{g}}{\int_{M} u_{\alpha}^{s} \mathrm{~d} v_{g}} .
$$

Noting that $\lim \int_{B(0, \delta)} \varphi_{\alpha}^{s} \mathrm{~d} v_{g_{\alpha}}>0$,

$$
\frac{\int_{B_{x_{\alpha}}\left(a_{\alpha} \delta\right)} u_{\alpha}^{s} \mathrm{~d} v_{g}}{\int_{M} u_{\alpha}^{s} \mathrm{~d} v_{g}} \geqslant C>0 .
$$

This ends the proof of Lemma 2.1.

One shows similarly that

$$
\left\|u_{\alpha}\right\|_{\infty}^{r-s} A_{\alpha}^{s \theta / 2(1-\theta)} \rightarrow C>0 .
$$

Let us note that (2.9) leads to $a_{\alpha}\left\|u_{\alpha}\right\|_{\infty}^{r / n} \rightarrow C>0$. As a consequence, $\left\|u_{\alpha}\right\|_{\infty} \rightarrow+\infty$ and $A_{\alpha} \rightarrow 0$. Moreover, since $s \leqslant 2$, we also have

$$
\int_{M} u_{\alpha}^{2} \mathrm{~d} v_{g} \leqslant \int_{M} u_{\alpha}^{s} \mathrm{~d} v_{g}\left\|u_{\alpha}\right\|_{\infty}^{2-s}=A_{\alpha}^{s \theta / 2(1-\theta)}\left\|u_{\alpha}\right\|_{\infty}^{2-s} .
$$

Consequently, by (2.9) and the inequality $\left\|u_{\alpha}\right\|_{\infty}^{2-r} \leqslant C \int_{M} u_{\alpha}^{2} \mathrm{~d} v_{g}$, we obtain

$$
\left\|u_{\alpha}\right\|_{\infty}^{r-2} \int_{M} u_{\alpha}^{2} \mathrm{~d} v_{g} \rightarrow C>0
$$

Remark. Relations (2.7) and (2.8) are intensively used throughout the proof and we will thus no longer be precise when they are needed.

One can now improve the previous lemma. Actually, we have the following.

Lemma 2.2. Let $\left(c_{\alpha}\right)_{\alpha}$ be a sequence of positive real numbers satisfying $\left(a_{\alpha} / c_{\alpha}\right) \rightarrow 0$. Then

$$
\lim \frac{\int_{B_{x_{\alpha}}\left(c_{\alpha}\right)} u_{\alpha}^{s} \mathrm{~d} v_{g}}{\int_{M} u_{\alpha}^{s} \mathrm{~d} v_{g}}=1 .
$$

Proof. Let $\eta \in C_{\infty}(\mathbb{R})$ be such that

(i) $\eta\left(\left[0, \frac{1}{2}\right]\right)=\{1\}$,

(ii) $\eta([1,+\infty])=\{0\}$,

(iii) $0 \leqslant \eta \leqslant 1$.

For $k \in \mathbb{N}$, set $\eta_{\alpha, k}=\left(\eta\left(c_{\alpha}^{-1} d_{g}\left(x, x_{\alpha}\right)\right)\right)^{2^{k}}$.

Multiplying (2.1) by $\eta_{\alpha, k}^{r} u_{\alpha}$ and integrating over $M$, we obtain

$$
\begin{aligned}
2 A_{\alpha} \int_{M} \eta_{\alpha, k}^{r} u_{\alpha} \Delta_{g} u_{\alpha} \mathrm{d} v_{g}+2 & \alpha A_{\alpha} \int_{M} \eta_{\alpha, k}^{r} u_{\alpha}^{2} \mathrm{~d} v_{g} \\
& +\frac{2(1-\theta)}{\theta} B_{\alpha} \int_{M} \eta_{\alpha, k}^{r} u_{\alpha}^{s} \mathrm{~d} v_{g}=k_{\alpha} \int_{M} \eta_{\alpha, k}^{r} u_{\alpha}^{r} \mathrm{~d} v_{g} .
\end{aligned}
$$


The identity

$$
\int_{M} \eta_{\alpha, k}^{r} u_{\alpha} \Delta_{g} u_{\alpha} \mathrm{d} v_{g}=\int_{M}\left|\nabla \eta_{\alpha, k}^{r / 2} u_{\alpha}\right|_{g}^{2} \mathrm{~d} v_{g}-\int_{M}\left|\nabla \eta_{\alpha, k}^{r / 2}\right|_{g}^{2} u_{\alpha}^{2} \mathrm{~d} v_{g}
$$

then leads to

$$
\begin{aligned}
2 A_{\alpha} \int_{M}\left|\nabla \eta_{\alpha, k}^{r / 2} u_{\alpha}\right|_{g}^{2} \mathrm{~d} v_{g}-2 A_{\alpha} \int_{M}\left|\nabla \eta_{\alpha, k}^{r / 2}\right|_{g}^{2} u_{\alpha}^{2} \mathrm{~d} v_{g}+2 \alpha A_{\alpha} \int_{M} \eta_{\alpha, k}^{r} u_{\alpha}^{2} \mathrm{~d} v_{g} \\
+\frac{2(1-\theta)}{\theta} B_{\alpha} \int_{M} \eta_{\alpha, k}^{r} u_{\alpha}^{s} \mathrm{~d} v_{g}=\frac{1}{2} k_{\alpha} \int_{M} \eta_{\alpha, k}^{r} u_{\alpha}^{r} \mathrm{~d} v_{g} .
\end{aligned}
$$

Moreover, (1.3) with $A=A_{0}+\epsilon, B=B_{\epsilon}$ and $u=\eta_{\alpha, k} u_{\alpha}$ gives

$$
\begin{aligned}
& \left(\int_{M}\left|\eta_{\alpha, k} u_{\alpha}\right|^{r} \mathrm{~d} v_{g}\right)^{2 / r \theta} \\
& \quad \leqslant\left(\left(A_{0}+\epsilon\right) \int_{M}\left|\nabla \eta_{\alpha, k} u_{\alpha}\right|_{g}^{2} \mathrm{~d} v_{g}+B_{\epsilon} \int_{M}\left|\eta_{\alpha, k} u_{\alpha}\right|^{2} \mathrm{~d} v_{g}\right)\left(\int_{M}\left|\eta_{\alpha, k} u_{\alpha}\right|^{s} \mathrm{~d} v_{g}\right)^{2(1-\theta) / s \theta}
\end{aligned}
$$

Set

$$
\begin{aligned}
\lambda_{k} & =\lim \frac{\int_{M} \eta_{\alpha, k}^{r} u_{\alpha}^{s} \mathrm{~d} v_{g}}{\int_{M} u_{\alpha}^{s} \mathrm{~d} v_{g}} \\
\tilde{\lambda}_{k} & =\lim \frac{\int_{M} \eta_{\alpha, k}^{s} u_{\alpha}^{s} \mathrm{~d} v_{g}}{\int_{M} u_{\alpha}^{s} \mathrm{~d} v_{g}} \\
X_{k} & =\lim A_{\alpha} \int_{M}\left|\nabla \eta_{\alpha, k}^{r / 2} u_{\alpha}\right|_{g}^{2} \mathrm{~d} v_{g}, \\
Y_{k} & =\lim A_{\alpha} \int_{M}\left|\nabla \eta_{\alpha, k} u_{\alpha}\right|_{g}^{2} \mathrm{~d} v_{g}, \\
Z_{k} & =\lim \int_{M} \eta_{\alpha, k}^{r} u_{\alpha}^{r} \mathrm{~d} v_{g} .
\end{aligned}
$$

Let us now search for some relations involving $\lambda_{k}, \tilde{\lambda}_{k}, X_{k}, Y_{k}$ and $Z_{k}$.

One has the following.

(i) Relation (2.10) implies that

$$
\lim A_{\alpha} \int_{M}\left|\nabla \eta_{\alpha, k}^{r / 2}\right|_{g}^{2} u_{\alpha}^{2} \mathrm{~d} v_{g} \leqslant \lim C \frac{a_{\alpha}^{2}}{c_{\alpha}^{2}}=0
$$

(ii) Relation (2.5) implies that

$$
\lim \alpha A_{\alpha} \int_{M} \eta_{\alpha, k}^{r} u_{\alpha}^{2} \mathrm{~d} v_{g}=0
$$


(iii) By definition of $A_{\alpha}$,

$$
\begin{aligned}
\lim \left(\int_{M}\left|\nabla \eta_{\alpha, k} u_{\alpha}\right|_{g}^{2} \mathrm{~d} v_{g}\right) & \left(\int_{M} \eta_{\alpha, k}^{r} u_{\alpha}^{s} \mathrm{~d} v_{g}\right)^{2(1-\theta) / s \theta} \\
= & \lim A_{\alpha}\left(\int_{M}\left|\nabla \eta_{\alpha, k} u_{\alpha}\right|_{g}^{2} \mathrm{~d} v_{g}\right) \tilde{\lambda}_{k}^{2(1-\theta) / s \theta} \\
& =Y_{k} \tilde{\lambda}_{k}^{2(1-\theta) / s \theta}
\end{aligned}
$$

and

$$
\lim \left(\int_{M} \eta_{\alpha, k}^{2} u_{\alpha}^{2} \mathrm{~d} v_{g}\right)\left(\int_{M} \eta_{\alpha, k}^{r} u_{\alpha}^{s} \mathrm{~d} v_{g}\right)^{2(1-\theta) / s \theta} \leqslant \lim A_{\alpha} \int_{M} u_{\alpha}^{2} \mathrm{~d} v_{g}=0 .
$$

Therefore, taking the limit in (2.11) and (2.12), we obtain

$$
\begin{gathered}
X_{k}+\frac{1-\theta}{\theta} \lambda_{k} A_{0}^{-1}=\frac{A_{0}}{\theta} Z_{k}, \\
Z_{k}^{2 / r \theta} \leqslant\left(A_{0}+\epsilon\right) Y_{k} \tilde{\lambda}_{k}^{2(1-\theta) / s \theta} .
\end{gathered}
$$

Set $\tilde{X}_{k}=A_{0} X_{k}$ and $\tilde{Y}_{k}=A_{0} Y_{k}$. Noting that $\epsilon$ is arbitrary, we then have

$$
\begin{gathered}
\theta \tilde{X}_{k}+(1-\theta) \lambda_{k}=Z_{k} \\
Z_{k}^{2 / r \theta} \leqslant \tilde{Y}_{k} \tilde{\lambda}_{k}^{2(1-\theta) / s \theta} .
\end{gathered}
$$

Now, let us remark that

$$
\lambda_{k}^{s}=\frac{\lambda_{k}^{s}}{\tilde{\lambda}_{k}^{r}} \tilde{\lambda}_{k}^{r}
$$

After some easy computations, it follows that

$$
\lambda_{k} \leqslant \frac{1}{1-\theta} \tilde{Y}_{k}^{r \theta / 2(1-\theta)}\left(Z_{k}^{1-(1 /(1-\theta))}-\theta \tilde{X}_{k} Z_{k}^{-1 /(1-\theta)}\right) \tilde{\lambda}_{k}^{r / s} .
$$

Set $f(x, z)=z^{1-(1 /(1-\theta))}-\theta x z^{-1 /(1-\theta)}$. One has

$$
\frac{\partial f}{\partial z}(x, z)=\frac{\theta}{1-\theta} z^{-1 /(1-\theta)}\left(\frac{x}{z}-1\right) .
$$

Since $\theta \tilde{X}_{k}+(1-\theta) \lambda_{k}=Z_{k}, \lambda_{k}<Z_{k}<\tilde{X}_{k}$ or $\tilde{X}_{k}<Z_{k}<\lambda_{k}$. In both cases, $f\left(\tilde{X}_{k}, Z_{k}\right)<$ $f\left(\tilde{X}_{k}, \tilde{X}_{k}\right)$. As a consequence,

$$
\lambda_{k} \leqslant\left(\tilde{Y}_{k}^{r / 2} \tilde{X}_{k}^{-1}\right)^{\theta /(1-\theta)} \tilde{\lambda}_{k}^{r}
$$

From Hölder's inequality for the measure $\mathrm{d} \mu_{\alpha}=\left|\nabla u_{\alpha}\right|_{g}^{2} \mathrm{~d} v_{g}$ and the equalities

$$
\begin{aligned}
& \tilde{Y}_{k}=\lim A_{0} A_{\alpha} \int_{M} \eta_{\alpha, k}^{2}\left|\nabla u_{\alpha}\right|_{g}^{2} \mathrm{~d} v_{g}, \\
& \tilde{X}_{k}=\lim A_{0} A_{\alpha} \int_{M} \eta_{\alpha, k}^{r}\left|\nabla u_{\alpha}\right|_{g}^{2} \mathrm{~d} v_{g},
\end{aligned}
$$


it follows that $\tilde{Y}_{k}^{r / 2} \leqslant \tilde{X}_{k}$ and $\lambda_{k} \leqslant \tilde{\lambda}_{k}^{r / s}$. Since, by Lemma 2.1,

$$
C \leqslant \lambda_{k+1} \leqslant \tilde{\lambda}_{k+1} \leqslant \lambda_{k} \leqslant \tilde{\lambda}_{k+1} \leqslant \lim \frac{\int_{B_{x_{\alpha}}\left(c_{\alpha}\right)} u_{\alpha}^{s} \mathrm{~d} v_{g}}{\int_{M} u_{\alpha}^{s} \mathrm{~d} v_{g}},
$$

we then have

$$
\forall N \in \mathbb{N}, \quad C \leqslant \lambda_{0}^{N r / s} \leqslant \lim \frac{\int_{B_{x_{\alpha}}\left(c_{\alpha}\right)} u_{\alpha}^{s} \mathrm{~d} v_{g}}{\int_{M} u_{\alpha}^{s} \mathrm{~d} v_{g}} .
$$

Thereafter,

$$
\lim \frac{\int_{B_{x_{\alpha}}\left(c_{\alpha}\right)} u_{\alpha}^{s} \mathrm{~d} v_{g}}{\int_{M} u_{\alpha}^{s} \mathrm{~d} v_{g}}=1
$$

and Lemma 2.2 is proved.

An important estimate follows.

Lemma 2.3. There exists $C>0$ independent of $\alpha$ such that for all $x \in M$ and every $\alpha$,

$$
u_{\alpha}(x) \mathrm{d}_{g}\left(x, x_{\alpha}\right)^{n / r} \leqslant C .
$$

Proof. Let us assume by contradiction that there exists a sequence $\left(y_{\alpha}\right)_{\alpha}$ of points of $M$ such that

$$
u_{\alpha}\left(y_{\alpha}\right) \mathrm{d}_{g}\left(y_{\alpha}, x_{\alpha}\right)^{n / r} \rightarrow+\infty .
$$

From now on, in most cases we set $r_{\alpha}=\mathrm{d}_{g}\left(\cdot, x_{\alpha}\right)$. Set $v_{\alpha}=u_{\alpha}\left(y_{\alpha}\right) \mathrm{d}_{g}\left(y_{\alpha}, x_{\alpha}\right)^{n / r}$. One can assume without loss of generality that $v_{\alpha}=\left\|u_{\alpha} r_{\alpha}^{n / r}\right\|_{\infty}$.

First, let us prove that for all $\nu$ small enough, we have

$$
B_{y_{\alpha}}\left(u_{\alpha}\left(y_{\alpha}\right)^{-r / n}\right) \cap B_{x_{\alpha}}\left(a_{\alpha} v_{\alpha}^{\nu}\right)=\emptyset .
$$

It is enough to prove $\mathrm{d}_{g}\left(y_{\alpha}, x_{\alpha}\right) \geqslant u_{\alpha}\left(y_{\alpha}\right)^{-r / n}+a_{\alpha} v_{\alpha}^{\nu}$ or, equivalently, $v_{\alpha}^{(r / n)-\nu} \geqslant v_{\alpha}^{-\nu}+$ $a_{\alpha} u_{\alpha}\left(y_{\alpha}\right)^{r / n}$. If $\nu<r / n$, we obtain from (2.13) that $v_{\alpha}^{(r / n)-\nu} \rightarrow \infty$ and $v_{\alpha}^{-\nu} \rightarrow 0$. One has yet to show that $v_{\alpha} u_{\alpha}\left(y_{\alpha}\right)^{r / n} \leqslant C$. Meanwhile, (2.9) implies

$$
\begin{aligned}
a_{\alpha} u_{\alpha}\left(y_{\alpha}\right)^{r / n} & \leqslant a_{\alpha}\left\|u_{\alpha}\right\|_{\infty}^{r / n} \\
& \leqslant\left(A_{\alpha}\left\|u_{\alpha}\right\|_{\infty}^{2-r}\left\|u_{\alpha}\right\|_{\infty}^{2(r / n)}\right)^{1 / 2} \\
& \leqslant C
\end{aligned}
$$

which proves (2.14).

Let us now set for all $x \in B(0,1)$

$$
\begin{aligned}
& h_{\alpha}(x)=\left(\exp _{y_{\alpha}}^{*} g\right)\left(l_{\alpha} x\right), \\
& \psi_{\alpha}(x)=u_{\alpha}\left(y_{\alpha}\right)^{-1} u_{\alpha}\left(\exp _{y_{\alpha}}\left(l_{\alpha} x\right)\right),
\end{aligned}
$$

where $l_{\alpha}=\left\|u_{\alpha}\right\|_{\infty}^{-((1 / 2)+(r / n))} u_{\alpha}\left(y_{\alpha}\right)^{1 / 2}$. 
From (2.1), one can easily check that

$$
\begin{aligned}
\Delta_{h_{\alpha}} \psi_{\alpha}(x) & =u_{\alpha}\left(y_{\alpha}\right)^{-1} l_{\alpha}^{2} \Delta_{g} u_{\alpha}\left(\exp _{y_{\alpha}}\left(l_{\alpha} x\right)\right) \\
& =\frac{k_{\alpha}\left\|u_{\alpha}\right\|_{\infty}^{-1-(2 r / n)} u_{\alpha}\left(y_{\alpha}\right)^{r-1}}{2 A_{\alpha}} \psi_{\alpha}(x)^{r-1}-\alpha\left\|u_{\alpha}\right\|_{\infty}^{-1-(2 r / n)} u_{\alpha}\left(y_{\alpha}\right) \psi_{\alpha}(x) \\
- & -\frac{(1-\theta) B_{\alpha}\left\|u_{\alpha}\right\|_{\infty}^{-1-(2 r / n)} u_{\alpha}\left(y_{\alpha}\right)^{s-1}}{\theta A_{\alpha}} \psi_{\alpha}(x)^{s-1} .
\end{aligned}
$$

Hence, under the assumption $\left\|\psi_{\alpha}\right\|_{L^{\infty}(B(0,1))} \leqslant C$ and by $(2.6)$,

$$
\begin{aligned}
\left|\Delta_{h_{\alpha}} \psi_{\alpha}(x)\right| & \leqslant C \frac{\left\|u_{\alpha}\right\|_{\infty}^{-1-(2 r / n)+r-1}}{2 A_{\alpha}} \\
& \leqslant C \frac{\left\|u_{\alpha}\right\|_{\infty}^{-(r-s)(2(1-\theta) / s \theta)}}{A_{\alpha}} \\
& \leqslant C .
\end{aligned}
$$

Let us now show that

$$
\left\|u_{\alpha}\right\|_{L_{\infty}\left(B_{y_{\alpha}}\left(l_{\alpha}\right)\right)} \leqslant\left\|u_{\alpha}\right\|_{L_{\infty}\left(B_{y_{\alpha}}\left(u_{\alpha}\left(y_{\alpha}\right)^{-r / n}\right)\right)} \leqslant C u_{\alpha}\left(y_{\alpha}\right) .
$$

By the definition of $y_{\alpha}$, we have for all $x \in B_{y_{\alpha}}\left(u_{\alpha}\left(y_{\alpha}\right)^{-r / n}\right)$,

$$
u_{\alpha}\left(y_{\alpha}\right) \mathrm{d}_{g}\left(y_{\alpha}, x_{\alpha}\right)^{n / r} \geqslant u_{\alpha}(x) \mathrm{d}_{g}\left(x, x_{\alpha}\right)^{n / r} .
$$

Moreover, since $x \in B_{y_{\alpha}}\left(u_{\alpha}\left(y_{\alpha}\right)^{-r / n}\right)$ and $u_{\alpha}\left(y_{\alpha}\right) \leqslant\left\|u_{\alpha}\right\|_{\infty}$, we have

$$
\mathrm{d}_{g}\left(x, y_{\alpha}\right) \leqslant u_{\alpha}\left(y_{\alpha}\right)^{-r / n}
$$

and by $(2.13), u_{\alpha}\left(y_{\alpha}\right)^{-r / n} \leqslant \frac{1}{2} \mathrm{~d}_{g}\left(y_{\alpha}, x_{\alpha}\right)$. Therefore,

$$
\mathrm{d}_{g}\left(x_{\alpha}, x\right) \geqslant \mathrm{d}_{g}\left(y_{\alpha}, x_{\alpha}\right)-\mathrm{d}_{g}\left(y_{\alpha}, x\right) \leqslant \mathrm{d}_{g}\left(y_{\alpha}, x_{\alpha}\right)-u_{\alpha}\left(y_{\alpha}\right)^{-r / n} \geqslant \frac{1}{2} \mathrm{~d}_{g}\left(y_{\alpha}, x_{\alpha}\right),
$$

which, combined with $(2.15)$, proves that

$$
\left\|u_{\alpha}\right\|_{L_{\infty}\left(B_{y_{\alpha}}\left(u_{\alpha}\left(y_{\alpha}\right)^{-r / n}\right)\right)} \leqslant C u_{\alpha}\left(y_{\alpha}\right) .
$$

Hence, we have $\left\|\psi_{\alpha}\right\|_{L_{\infty}\left(B_{y_{\alpha}}\left(l_{\alpha}\right)\right)} \leqslant C$ and, as a consequence, $\left\|\Delta_{h_{\alpha}} \psi_{\alpha}\right\|_{L_{\infty}\left(B_{y_{\alpha}}\left(l_{\alpha}\right)\right)} \leqslant C$. By arguments already used above, there exists $\psi \in C^{0}(B(0,1))$ such that $\psi_{\alpha} \rightarrow \psi$ in $C^{0}(B(0,1))$ with $\psi(0)>0$. One then has

$$
\begin{aligned}
\int_{B(0,1)} \psi_{\alpha}^{s} \mathrm{~d} v_{h_{\alpha}} & =A_{\alpha}^{s \theta / 2(1-\theta)} u_{\alpha}\left(y_{\alpha}\right)^{-s} l_{\alpha}^{-n} \frac{\int_{B_{y_{\alpha}}\left(l_{\alpha}\right)} u_{\alpha}^{s} \mathrm{~d} v_{g}}{\int_{M} u_{\alpha}^{s} \mathrm{~d} v_{g}} \\
& \stackrel{+\infty}{\sim} C\left(\frac{\left\|u_{\alpha}\right\|_{\infty}}{u_{\alpha}\left(y_{\alpha}\right)}\right)^{(n / 2)+s} \frac{\int_{B_{y_{\alpha}}\left(l_{\alpha}\right)} u_{\alpha}^{s} \mathrm{~d} v_{g}}{\int_{M} u_{\alpha}^{s} \mathrm{~d} v_{g}} .
\end{aligned}
$$


Set

We obtain

$$
m_{\alpha}=\frac{u_{\alpha}\left(y_{\alpha}\right)}{\left\|u_{\alpha}\right\|_{\infty}}
$$

$$
\frac{\int_{B_{y_{\alpha}}\left(l_{\alpha}\right)} u_{\alpha}^{s} \mathrm{~d} v_{g}}{\int_{M} u_{\alpha}^{s} \mathrm{~d} v_{g}} \stackrel{+\infty}{\sim} C m_{\alpha}^{(n / 2)+s} .
$$

Lemma 2.2 and (2.14) imply

$$
\lim \frac{\int_{B_{y_{\alpha}}\left(u_{\alpha}\left(y_{\alpha}\right)^{-r / n}\right)} u_{\alpha}^{s} \mathrm{~d} v_{g}}{\int_{M} u_{\alpha}^{s} \mathrm{~d} v_{g}}=0 .
$$

Consequently, $\lim m_{\alpha}=0$. Now, let us show that there exists a sequence $\left(\gamma_{k}\right)_{k>0}$ of positive real numbers converging to $+\infty$ such that for all $k>0$,

$$
m_{\alpha}^{-\gamma_{k}} \int_{B_{y_{\alpha}}\left(2^{-k} u_{\alpha}\left(y_{\alpha}\right)^{-r / n}\right)} u_{\alpha}^{r} \mathrm{~d} v_{g} \rightarrow 0 .
$$

Let us proceed by induction. Since $\left\|u_{\alpha}\right\|_{L_{\infty}\left(B_{y_{\alpha}}\left(u_{\alpha}\left(y_{\alpha}\right)^{-r / n}\right)\right)} \leqslant C u_{\alpha}\left(y_{\alpha}\right)$, we have

$$
\begin{aligned}
\int_{B_{y_{\alpha}}\left(u_{\alpha}\left(y_{\alpha}\right)^{-r / n}\right)} u_{\alpha}^{r} \mathrm{~d} v_{g} & \leqslant C u_{\alpha}\left(y_{\alpha}\right)^{r-s} \int_{B_{y_{\alpha}}\left(u_{\alpha}\left(y_{\alpha}\right)^{-r / n}\right)} u_{\alpha}^{s} \mathrm{~d} v_{g} \\
& \leqslant C m_{\alpha}^{r-s}\left\|u_{\alpha}\right\|_{\infty}^{r-s} \int_{B_{y_{\alpha}}\left(u_{\alpha}\left(y_{\alpha}\right)^{-r / n}\right)} u_{\alpha}^{s} \mathrm{~d} v_{g} .
\end{aligned}
$$

Therefore, we can set $\gamma_{0}=r-s$ by (2.14). Let us assume that we constructed the sequence up to some $k>0$.

Set $\eta_{\alpha, k}(x)=\eta\left(2^{k} u_{\alpha}\left(y_{\alpha}\right)^{r / n} d_{g}\left(y_{\alpha}, x\right)\right)$.

Multiplying (2.1) by $u_{\alpha} \eta_{\alpha, k}^{2} / m_{\alpha}^{\gamma_{k}}$ and integrating over $M$, we obtain

$$
\begin{aligned}
\frac{2 A_{\alpha}}{m_{\alpha}^{\gamma_{k}}} \int_{M}\left|\nabla \eta_{\alpha, k} u_{\alpha}\right|_{g}^{2} \mathrm{~d} v_{g}-\frac{2 A_{\alpha}}{m_{\alpha}^{\gamma_{k}}} \int_{M}\left|\nabla \eta_{\alpha, k}\right|_{g}^{2} u_{\alpha}^{2} \mathrm{~d} v_{g}+\frac{2 \alpha A_{\alpha}}{m_{\alpha}^{\gamma_{k}}} \int_{M} \eta_{\alpha, k}^{2} u_{\alpha}^{2} \mathrm{~d} v_{g} \\
+\frac{2(1-\theta)}{\theta} \frac{B_{\alpha}}{m_{\alpha}^{\gamma_{k}}} \int_{M} \eta_{\alpha, k}^{2} u_{\alpha}^{s} \mathrm{~d} v_{g}=\frac{k_{\alpha}}{m_{\alpha}^{\gamma_{k}}} \int_{M} \eta_{\alpha, k}^{2} u_{\alpha}^{r}, \mathrm{~d} v_{g} .
\end{aligned}
$$

Relation (2.16) and Hölder's inequality imply

$$
\begin{aligned}
A_{\alpha} m_{\alpha}^{-\gamma_{k}} \int_{M}\left|\nabla \eta_{\alpha, k}\right|_{g}^{2} u_{\alpha}^{2} \mathrm{~d} v_{g} & \leqslant C A_{\alpha} u_{\alpha}\left(y_{\alpha}\right)^{2 r / n} m_{\alpha}^{-\gamma_{k}} \int_{B_{y_{\alpha}}\left(2^{-k} u_{\alpha}\left(y_{\alpha}\right)^{-r / n}\right)} u_{\alpha}^{2} \mathrm{~d} v_{g} \\
& \leqslant C\left\|u_{\alpha}\right\|_{\infty}^{r-2} m_{\alpha}^{2 r / n} m_{\alpha}^{-\gamma_{k}} \int_{B_{y_{\alpha}}\left(2^{-k} u_{\alpha}\left(y_{\alpha}\right)^{-r / n}\right)} u_{\alpha}^{2} \mathrm{~d} v_{g} \\
& \leqslant C\left\|u_{\alpha}\right\|_{\infty}^{r-2} m_{\alpha}^{2 r / n} m_{\alpha}^{-\gamma_{k}}\left(\operatorname{Vol}_{g}\left(B_{y_{\alpha}}\left(2^{-k} u_{\alpha}\left(y_{\alpha}\right)^{-r / n}\right)\right)\right)^{1-(2 / r)} \\
& \times\left(\int_{B_{y_{\alpha}}\left(2^{-k} u_{\alpha}\left(y_{\alpha}\right)^{-r / n}\right)} u_{\alpha}^{r} \mathrm{~d} v_{g}\right)^{2 / r} \\
& \leqslant C m_{\alpha}^{2-r+(2 r / n)-\gamma_{k}+(2 / r) \gamma_{k}} \\
& \leqslant C m_{\alpha}^{(r-s)(2(1-\theta) / s \theta)-\gamma_{k}(1-(2 / r))}
\end{aligned}
$$


and

$$
k_{\alpha} m_{\alpha}^{-\gamma_{k}} \int_{M} \eta_{\alpha, k}^{2} u_{\alpha}^{r} \mathrm{~d} v_{g} \leqslant C .
$$

There are now two possibilities. One is the case

$$
(r-s) \frac{2(1-\theta)}{s \theta}-\gamma_{k}\left(1-\frac{2}{r}\right) \geqslant 0
$$

One then has, by (2.17),

$$
\begin{array}{r}
A_{\alpha} m_{\alpha}^{-\gamma_{k}} \int_{M} \eta_{\alpha, k}^{2} u_{\alpha}^{2} \mathrm{~d} v_{g} \leqslant C, \\
B_{\alpha} m_{\alpha}^{-\gamma_{k}} \int_{M} \eta_{\alpha, k}^{2} u_{\alpha}^{s} \mathrm{~d} v_{g} \leqslant C, \\
A_{\alpha} m_{\alpha}^{-\gamma_{k}} \int_{M}\left|\nabla \eta_{\alpha, k} u_{\alpha}\right|_{g}^{2} \mathrm{~d} v_{g} \leqslant C .
\end{array}
$$

Moreover, we obtain from (1.3) with $u=\eta_{\alpha} u_{\alpha}$ that

$$
\begin{aligned}
\left(\int_{M} \eta_{\alpha, k}^{r} u_{\alpha}^{r} \mathrm{~d} v_{g}\right)^{2 / r \theta} \leqslant A \int_{M}\left|\nabla \eta_{\alpha, k} u_{\alpha}\right|_{g}^{2} \mathrm{~d} v_{g}\left(\int_{M} \eta_{\alpha, k}^{s} u_{\alpha}^{s} \mathrm{~d} v_{g}\right)^{2(1-\theta) / s \theta} & \\
& +B \int_{M} \eta_{\alpha, k}^{2} u_{\alpha}^{2} \mathrm{~d} v_{g}\left(\int_{M} \eta_{\alpha, k}^{s} u_{\alpha}^{s} \mathrm{~d} v_{g}\right)^{2(1-\theta) / s \theta} .
\end{aligned}
$$

Noting that (2.18) is still valid by changing $\eta$ into $\eta^{s / 2}$, we then have

$$
\begin{aligned}
\int_{M}\left|\nabla \eta_{\alpha, k} u_{\alpha}\right|_{g}^{2} \mathrm{~d} v_{g}\left(\int_{M} \eta_{\alpha, k}^{s} u_{\alpha}^{s} \mathrm{~d} v_{g}\right)^{2(1-\theta) / s \theta} & \\
& \leqslant \frac{C}{A_{\alpha} B_{\alpha}^{2(1-\theta) / s \theta}} A_{\alpha} \int_{M}\left|\nabla \eta_{\alpha, k} u_{\alpha}\right|_{g}^{2} \mathrm{~d} v_{g}\left(B_{\alpha} \int_{M} \eta_{\alpha, k}^{s} u_{\alpha}^{s} \mathrm{~d} v_{g}\right)^{2(1-\theta) / s \theta} \\
& \leqslant C m_{\alpha}^{(1+(2(1-\theta) / s \theta)) \gamma_{k}}
\end{aligned}
$$

and

$$
\begin{aligned}
\int_{M} \eta_{\alpha, k}^{2} u_{\alpha}^{2} \mathrm{~d} & v_{g}\left(\int_{M} \eta_{\alpha, k}^{s} u_{\alpha}^{s} \mathrm{~d} v_{g}\right)^{2(1-\theta) / s \theta} \\
& \leqslant \frac{C}{B_{\alpha}^{2(1-\theta) / s \theta}} \int_{M} \eta_{\alpha, k}^{2} u_{\alpha}^{2} \mathrm{~d} v_{g}\left(B_{\alpha} \int_{M} \eta_{\alpha, k}^{s} u_{\alpha}^{s} \mathrm{~d} v_{g}\right)^{2(1-\theta) / s \theta} \\
& \leqslant C m_{\alpha}^{(1+(2(1-\theta) / s \theta)) \gamma_{k}} .
\end{aligned}
$$

Thereafter, by using the relation

$$
\int_{B_{y_{\alpha}}\left(2^{-(k+1)} u_{\alpha}\left(y_{\alpha}\right)^{-r / n}\right)} u_{\alpha}^{r} \mathrm{~d} v_{g} \leqslant \int_{M} \eta_{\alpha, k}^{r} u_{\alpha}^{r} \mathrm{~d} v_{g}
$$


we obtain

$$
\begin{aligned}
\int_{B_{y_{\alpha}}\left(2^{-(k+1)} u_{\alpha}\left(y_{\alpha}\right)^{-r / n}\right)} u_{\alpha}^{r} \mathrm{~d} v_{g} & \leqslant C m_{\alpha}^{(r \theta / 2)(1+(2(1-\theta) / s \theta)) \gamma_{k}} \\
& \leqslant C m_{\alpha}^{((r \theta / n)+1) \gamma_{k}} .
\end{aligned}
$$

Consequently, we can set $\gamma_{k+1}=((r \theta / 2 n)+1) \gamma_{k}$.

The other possibility is

$$
(r-s) \frac{2(1-\theta)}{s \theta}-\gamma_{k}\left(1-\frac{2}{r}\right)<0 .
$$

The same arguments as above give

$$
\int_{B_{y_{\alpha}}\left(2^{-(k+1)} u_{\alpha}\left(y_{\alpha}\right)^{-r / n}\right)} u_{\alpha}^{r} \mathrm{~d} v_{g} \leqslant C m_{\alpha}^{(r \theta / 2)(1+(2(1-\theta) / s \theta))\left((r-s)(2(1-\theta) / s \theta)+(2 / r) \gamma_{k}\right)}
$$

and

$$
\begin{array}{r}
m_{\alpha}^{-\gamma_{k}} \int_{B_{y_{\alpha}}\left(2^{-(k+1)} u_{\alpha}\left(y_{\alpha}\right)^{-r / n}\right)} u_{\alpha}^{r} \mathrm{~d} v_{g} \\
\leqslant C m_{\alpha}^{(r \theta / 2)(1+(2(1-\theta) / s \theta))((r-s)(2(1-\theta) / s \theta))+\gamma_{k}((r \theta / 2)(1+(2(1-\theta) / s \theta))(2 / r)-1)} .
\end{array}
$$

Thereafter, the relation

$$
\begin{aligned}
\frac{r \theta}{2}\left(1+\frac{2(1-\theta)}{s \theta}\right) \frac{2}{r}-1 & =\frac{r \theta}{2} \frac{2(1-\theta)}{s \theta}\left(\frac{2}{r}\left(\frac{s \theta}{2(1-\theta)}+1\right)-\frac{2}{r \theta} \frac{s \theta}{2(1-\theta)}\right) \\
& =\frac{r \theta}{2} \frac{2(1-\theta)}{s \theta} \frac{2-s}{r} \geqslant 0
\end{aligned}
$$

implies

$$
m_{\alpha}^{-\gamma_{k}} \int_{B_{y_{\alpha}}\left(2-(k+1) u_{\alpha}\left(y_{\alpha}\right)^{-r / n}\right)} u_{\alpha}^{r} \mathrm{~d} v_{g} \leqslant C m_{\alpha}^{(r \theta / 2)(1+(2(1-\theta) / s \theta))((r-s)(2(1-\theta) / s \theta)) .}
$$

Since

$$
\frac{r \theta}{2}\left(1+\frac{2(1-\theta)}{s \theta}\right)>1
$$

set $\gamma_{k+1}=\gamma_{k}+(r-s)(2(1-\theta) / s \theta)$. One can easily check that the sequence $\left(\gamma_{k}\right)_{k>0}$ converges to $+\infty$. Since $l_{\alpha} u_{\alpha}\left(y_{\alpha}\right)^{r / n} \rightarrow 0$, we have also proved that for all $k>0$,

$$
m_{\alpha}^{-\gamma_{k}} \int_{B_{y_{\alpha}}\left(l_{\alpha}\right)} u_{\alpha}^{r} \mathrm{~d} v_{g} \rightarrow 0 .
$$

But since

$$
\int_{B(0,1)} \psi_{\alpha}^{r} \mathrm{~d} v_{h_{\alpha}}=u_{\alpha}\left(y_{\alpha}\right)^{-r} l_{\alpha}^{-n} \int_{B_{y_{\alpha}}\left(l_{\alpha}\right)} u_{\alpha}^{r} \mathrm{~d} v_{g}
$$


we also have

$$
\int_{B_{y_{\alpha}}\left(l_{\alpha}\right)} u_{\alpha}^{r} \mathrm{~d} v_{g} \stackrel{+\infty}{\sim} C m_{\alpha}^{(n / 2)+r} .
$$

This leads to a contradiction and this ends the proof of Lemma 2.3.

Let $c>0$. Before concluding, we need some sharp estimates. The first one is the following.

Lemma 2.4. If $r \neq 2$, there exists $C>0$ independent of $\alpha$ such that

$$
A_{\alpha}^{-r /(r-2)} \int_{M-B_{x_{\alpha}}(c)} u_{\alpha}^{r} \mathrm{~d} v_{g} \leqslant C .
$$

If $r=2$, for all $k>0$, there exists $C>0$ independent of $\alpha$ such that

$$
A_{\alpha}^{-k} \int_{M-B_{x_{\alpha}}(c)} u_{\alpha}^{r} \mathrm{~d} v_{g} \leqslant C
$$

Proof. One starts with the case $r \neq 2$. Let $\delta \in] 0,(s \theta / 2(1-\theta))[$. Lemma 2.3 gives

$$
\begin{aligned}
A_{\alpha}^{-\delta} \int_{M-B_{x_{\alpha}}(c)} u_{\alpha}^{r} \mathrm{~d} v_{g} & \leqslant C A_{\alpha}^{-\delta} \int_{M-B_{x_{\alpha}}(c)} u_{\alpha}^{s} r_{\alpha}^{n(r-s) / r} \mathrm{~d} v_{g} \\
& \leqslant C A_{\alpha}^{-\delta} \int_{M-B_{x_{\alpha}}(c)} u_{\alpha}^{s} \mathrm{~d} v_{g} \\
& \leqslant C A_{\alpha}^{-\delta} A_{\alpha}^{s \theta / 2(1-\theta)} .
\end{aligned}
$$

Hence,

$$
A_{\alpha}^{-\delta} \int_{M-B_{x_{\alpha}}(c)} u_{\alpha}^{r} \mathrm{~d} v_{g} \rightarrow 0 .
$$

Let us show by induction that for all $k_{0}+1 \geqslant k>0$,

$$
A_{\alpha}^{-\delta((r \theta / 2 n)+1)^{k}} \int_{M-B_{x_{\alpha}}\left(2^{k} c\right)} u_{\alpha}^{r} \mathrm{~d} v_{g} \leqslant C
$$

where $k_{0}$ is such that

$$
\delta\left(\frac{r \theta}{2 n}+1\right)^{k_{0}} \leqslant \frac{r}{r-2} .
$$

Set $\eta_{\alpha, k}(x)=1-\eta\left(2^{-k} c^{-1} d_{g}\left(x_{\alpha}, x\right)\right)$ and $\epsilon_{k}=((r \theta / 2 n)+1)^{k}$. Assume that $(2.20)$ is true for some $k \leqslant k_{0}$. Multiplying (2.1) by $u_{\alpha} \eta_{\alpha, k}^{2} / A_{\alpha}^{\delta \epsilon_{k}}$ and integrating over $M$, we then obtain

$$
\begin{aligned}
& \frac{2 A_{\alpha}}{A_{\alpha}^{\delta \epsilon_{k}}} \int_{M}\left|\nabla \eta_{\alpha, k} u_{\alpha}\right|_{g}^{2} \mathrm{~d} v_{g}-\frac{2 A_{\alpha}}{A_{\alpha}^{\delta \epsilon_{k}}} \int_{M}\left|\nabla \eta_{\alpha, k}\right|_{g}^{2} u_{\alpha}^{2} \mathrm{~d} v_{g} \\
& \quad+\frac{2 \alpha A_{\alpha}}{A_{\alpha}^{\delta \delta_{k}}} \int_{M} \eta_{\alpha, k}^{2} u_{\alpha}^{2} \mathrm{~d} v_{g}+\frac{2(1-\theta)}{\theta} \frac{B_{\alpha}}{A_{\alpha}^{\delta \epsilon_{k}}} \int_{M} \eta_{\alpha, k}^{2} u_{\alpha}^{s} \mathrm{~d} v_{g}=\frac{k_{\alpha}}{A_{\alpha}^{\delta \epsilon_{k}}} \int_{M} \eta_{\alpha, k}^{2} u_{\alpha}^{r} \mathrm{~d} v_{g} .
\end{aligned}
$$


Since $\delta \epsilon_{k} \leqslant(r /(r-2))$, we have, by Hölder's inequality and (2.20),

$$
\begin{aligned}
A_{\alpha}^{1-\delta \epsilon_{k}} \int_{M}\left|\nabla \eta_{\alpha, k}\right|_{g}^{2} u_{\alpha}^{2} \mathrm{~d} v_{g} & \leqslant C A_{\alpha}^{1-\delta \epsilon_{k}(1-(2 / r))}\left(A_{\alpha}^{-\delta \epsilon_{k}} \int_{M-B_{x_{\alpha}}\left(2^{-k} c\right)} u_{\alpha}^{r} \mathrm{~d} v_{g}\right)^{2 / r} \\
& \leqslant C
\end{aligned}
$$

and

$$
\frac{k_{\alpha}}{A_{\alpha}^{\delta \epsilon_{k}}} \int_{M} \eta_{\alpha, k}^{2} u_{\alpha}^{r} \mathrm{~d} v_{g} \leqslant C
$$

Hence, by (2.21),

$$
\left.\begin{array}{r}
\frac{2 A_{\alpha}}{A_{\alpha}^{\delta \epsilon_{k}}} \int_{M}\left|\nabla \eta_{\alpha, k} u_{\alpha}\right|_{g}^{2} \mathrm{~d} v_{g} \leqslant C, \\
\frac{2 \alpha A_{\alpha}}{A_{\alpha}^{\delta \epsilon_{k}}} \int_{M} \eta_{\alpha, k}^{2} u_{\alpha}^{2} \mathrm{~d} v_{g} \leqslant C, \\
\frac{2(1-\theta)}{\theta} \frac{B_{\alpha}}{A_{\alpha}^{\delta \epsilon_{k}}} \int_{M} \eta_{\alpha, k}^{2} u_{\alpha}^{s} \mathrm{~d} v_{g} \leqslant C .
\end{array}\right\}
$$

Moreover, (1.3) with $u=\eta_{\alpha, k} u_{\alpha}$ gives

$$
\begin{aligned}
\left(\int_{M} \eta_{\alpha, k}^{r} u_{\alpha}^{r} \mathrm{~d} v_{g}\right)^{2 / r \theta} \leqslant A \int_{M}\left|\nabla \eta_{\alpha, k} u_{\alpha}\right|_{g}^{2} \mathrm{~d} v_{g}\left(\int_{M} \eta_{\alpha, k}^{s} u_{\alpha}^{s} \mathrm{~d} v_{g}\right)^{2(1-\theta) / s \theta} & \\
& +B \int_{M} \eta_{\alpha, k}^{2} u_{\alpha}^{2} \mathrm{~d} v_{g}\left(\int_{M} \eta_{\alpha, k}^{s} u_{\alpha}^{s} \mathrm{~d} v_{g}\right)^{2(1-\theta) / s \theta} .
\end{aligned}
$$

Noting that (2.22) is still valid after changing $\eta$ into $\eta^{s / 2}$, we then have

$$
\begin{aligned}
\int_{M}\left|\nabla \eta_{\alpha, k} u_{\alpha}\right|_{g}^{2} \mathrm{~d} v_{g}\left(\int_{M} \eta_{\alpha, k}^{s} u_{\alpha}^{s} \mathrm{~d} v_{g}\right)^{2(1-\theta) / s \theta} \\
\leqslant \frac{C}{A_{\alpha} B_{\alpha}^{2(1-\theta) / s \theta}} A_{\alpha} \int_{M}\left|\nabla \eta_{\alpha, k} u_{\alpha}\right|_{g}^{2} \mathrm{~d} v_{g}\left(B_{\alpha} \int_{M} \eta_{\alpha, k}^{s} u_{\alpha}^{s} \mathrm{~d} v_{g}\right)^{2(1-\theta) / s \theta} \\
\leqslant C A_{\alpha}^{(1+(2(1-\theta) / s \theta)) \delta \epsilon_{k}}
\end{aligned}
$$

and

$$
\begin{aligned}
\int_{M} \eta_{\alpha, k}^{2} u_{\alpha}^{2} \mathrm{~d} v_{g} & \left(\int_{M} \eta_{\alpha, k}^{s} u_{\alpha}^{s} \mathrm{~d} v_{g}\right)^{2(1-\theta) / s \theta} \\
& \leqslant \frac{C}{B_{\alpha}^{2(1-\theta) / s \theta}} \int_{M} \eta_{\alpha, k}^{2} u_{\alpha}^{2} \mathrm{~d} v_{g}\left(B_{\alpha} \int_{M} \eta_{\alpha, k}^{s} u_{\alpha}^{s} \mathrm{~d} v_{g}\right)^{2(1-\theta) / s \theta} \\
& \leqslant C A_{\alpha}^{(1+(2(1-\theta) / s \theta)) \delta \epsilon_{k}} .
\end{aligned}
$$

Thereafter,

$$
\left(\int_{M} \eta_{\alpha, k}^{r} u_{\alpha}^{r} \mathrm{~d} v_{g}\right)^{2 / r \theta} \leqslant C A_{\alpha}^{(1+(2(1-\theta) / s \theta)) \delta \epsilon_{k}}
$$


Hence, from the inequality

$$
\int_{M-B_{x_{\alpha}}\left(2^{k+1} c\right)} u_{\alpha}^{r} \mathrm{~d} v_{g} \leqslant \int_{M} \eta_{\alpha, k}^{r} u_{\alpha}^{r} \mathrm{~d} v_{g}
$$

we obtain

$$
\int_{M-B_{x_{\alpha}}\left(2^{k+1} c\right)} u_{\alpha}^{r} \mathrm{~d} v_{g} \leqslant C A_{\alpha}^{(1+(2(1-\theta) / s \theta)) \delta(r \theta / 2) \epsilon_{k}}
$$

Since

$$
\left(1+\frac{2(1-\theta)}{s \theta}\right) \frac{r \theta}{2}=\frac{r \theta}{n}+1>\frac{r \theta}{2 n}+1,
$$

we deduce (2.20) with rank $k+1$.

Let us remark that we have not only proved (2.19) but similarly, by a last induction, we have shown that

$$
A_{\alpha}^{-(r /(r-2))+(s \theta / 2(1-\theta))} \int_{M-B_{x_{\alpha}}(c)} u_{\alpha}^{s} \mathrm{~d} v_{g} \leqslant C .
$$

The case $r=2$ is handled identically, except that the induction can be continued forever.

In order to prove Lemma 2.6, we first have to show the following.

Lemma 2.5. There exists $t_{0}>0$ such that

$$
\forall x \in M-B_{x_{\alpha}}\left(t_{0} A_{\alpha}^{r s \theta / 2 n(r-s)(1-\theta)}\right), \quad \Delta_{g} u_{\alpha}(x)<0 .
$$

Proof. Let $x \in M$ be such that $\Delta_{g} u_{\alpha}(x)>0$. One then has, by (2.1), that

$$
\alpha A_{\alpha}+\frac{1-\theta}{\theta} B_{\alpha} u_{\alpha}(x)^{s-2} \leqslant \frac{1}{2} k_{\alpha} u_{\alpha}(x)^{r-2} .
$$

Hence, $C B_{\alpha} \leqslant u_{\alpha}(x)^{r-s}$. Moreover, by (2.3), we have $B_{\alpha} \geqslant C A_{\alpha}^{-s \theta / 2(1-\theta)}$. Hence,

$$
u_{\alpha}(x) \geqslant C A_{\alpha}^{-s \theta / 2(r-s)(1-\theta)} .
$$

By using Lemma 2.3, which gives $u_{\alpha}(x) \leqslant C r_{\alpha}^{-n / r}$, we obtain

$$
\mathrm{d}_{g}\left(x, x_{\alpha}\right) \geqslant C A_{\alpha}^{r s \theta / 2 n(r-s)(1-\theta)} .
$$

This proves our assertion.

In order to simplify the notation, set $\omega=r s \theta / 2 n(r-s)(1-\theta)$. Set $\eta_{\alpha}=\eta\left(c^{-1} r_{\alpha}\right)$. One can now prove the following.

Lemma 2.6. There exists $C>0$ independent of $\alpha$ such that

$$
\int_{M} \eta_{\alpha}^{2} r_{\alpha}^{2}\left|\nabla u_{\alpha}\right|_{g}^{2} \mathrm{~d} v_{g} \leqslant C\left\|u_{\alpha}\right\|_{\infty}^{2-r}
$$


Proof. Set $\gamma_{\alpha}=\int_{M} \eta_{\alpha, k}^{2} r_{\alpha}^{2}\left|\nabla u_{\alpha}\right|_{g}^{2} \mathrm{~d} v_{g}$. Integrating by parts, we obtain

$$
\gamma_{\alpha}=\int_{M} \eta_{\alpha}^{2} r_{\alpha}^{2} u_{\alpha} \Delta_{g} u_{\alpha} \mathrm{d} v_{g}-2 \int_{M} u_{\alpha} \eta_{\alpha} r_{\alpha}\left\langle\nabla u_{\alpha}, \nabla \eta_{\alpha} r_{\alpha}\right\rangle_{g} \mathrm{~d} v_{g} .
$$

Hence, by Lemma 2.5,

$$
\gamma_{\alpha} \leqslant \int_{B_{x_{\alpha}}\left(t_{0} A_{\alpha}^{\omega}\right)} \eta_{\alpha}^{2} r_{\alpha}^{2} u_{\alpha} \Delta_{g} u_{\alpha} \mathrm{d} v_{g}+C \int_{M} u_{\alpha} \eta_{\alpha} r_{\alpha}\left|\nabla u_{\alpha}\right|_{g}\left|\nabla \eta_{\alpha} r_{\alpha}\right|_{g} \mathrm{~d} v_{g} .
$$

Relations (2.1), (2.6) and (2.9) give

$$
\begin{aligned}
\left|u_{\alpha} \Delta_{g} u_{\alpha}\right| & \leqslant \frac{1}{2 A_{\alpha}}\left|k_{\alpha} u_{\alpha}^{r}-2 \alpha A_{\alpha} u_{\alpha}^{2}-\frac{2(1-\theta)}{\theta} B_{\alpha} u_{\alpha}^{s}\right| \\
& \leqslant C \frac{k_{\alpha}}{2 A_{\alpha}}\left\|u_{\alpha}\right\|_{\infty}^{r} \leqslant A_{\alpha}^{-n \omega-1} .
\end{aligned}
$$

It follows that

$$
\begin{aligned}
\int_{B_{x_{\alpha}}\left(t_{0} A_{\alpha}^{\omega}\right)} \eta_{\alpha}^{2} r_{\alpha}^{2} u_{\alpha} \Delta_{g} u_{\alpha} \mathrm{d} v_{g} & \leqslant C \operatorname{Vol}_{g}\left(B_{x_{\alpha}}\left(t_{0} A_{\alpha}^{\omega}\right)\right) A_{\alpha}^{-n \omega-1}\left(t_{0} A_{\alpha}^{\omega}\right)^{2} \\
& \leqslant C A_{\alpha}^{2 \omega-1}
\end{aligned}
$$

One may easily check that

$$
2 \omega-1=\frac{r s \theta}{n(r-s)(1-\theta)}-1=\frac{s \theta}{2(r-s)(1-\theta)}(r-2) .
$$

Hence

$$
\begin{aligned}
\int_{B_{x_{\alpha}}\left(t_{0} A_{\alpha}^{\omega}\right)} \eta_{\alpha}^{2} r_{\alpha}^{2} u_{\alpha} \Delta_{g} u_{\alpha} \mathrm{d} v_{g} & \leqslant C A_{\alpha}^{(s \theta /(2(r-s)(1-\theta)))(r-2)} \\
& \leqslant C\left\|u_{\alpha}\right\|_{\infty}^{2-r} .
\end{aligned}
$$

Moreover, Hölder's inequality leads to

$$
\int_{M} u_{\alpha} \eta_{\alpha} r_{\alpha}\left|\nabla u_{\alpha}\right|_{g}\left|\nabla \eta_{\alpha} r_{\alpha}\right|_{g} \mathrm{~d} v_{g} \leqslant\left(\int_{M} \eta_{\alpha}^{r} r_{\alpha}^{r}\left|\nabla u_{\alpha}\right|_{g}^{2} \mathrm{~d} v_{g}\right)^{1 / 2}\left(\int_{M} u_{\alpha}^{2}\left|\nabla \eta_{\alpha} r_{\alpha}\right|_{g}^{2} \mathrm{~d} v_{g}\right)^{1 / 2} .
$$

But

$$
\left|\nabla \eta_{\alpha} r_{\alpha}\right|_{g}^{2} \leqslant C
$$

Therefore,

$$
\int_{M} u_{\alpha} \eta_{\alpha} r_{\alpha}\left|\nabla u_{\alpha}\right|_{g}\left|\nabla \eta_{\alpha} r_{\alpha}\right|_{g} \mathrm{~d} v_{g} \leqslant\left(\gamma_{\alpha}\left\|u_{\alpha}\right\|_{\infty}^{2-r}\right)^{1 / 2}
$$

One then has

$$
\frac{\gamma_{\alpha}}{\left\|u_{\alpha}\right\|_{\infty}^{2-r}} \leqslant C+C\left(\frac{\gamma_{\alpha}}{\left\|u_{\alpha}\right\|_{\infty}^{2-r}}\right)^{1 / 2}
$$

which proves the lemma. 
Changing $\eta$ into $\eta^{r / 2}$, we also obtain

$$
\int_{M} \eta_{\alpha}^{r} r_{\alpha}^{2}\left|\nabla u_{\alpha}\right|_{g}^{2} \mathrm{~d} v_{g} \leqslant C\left\|u_{\alpha}\right\|_{\infty}^{2-r}
$$

We now prove the following main estimate.

Lemma 2.7. There exists $C>0$ independent of $\alpha$ such that

$$
\int_{M} u_{\alpha}^{r} \eta_{\alpha}^{r} r_{\alpha}^{2} \mathrm{~d} v_{g} \leqslant C \sqrt{\alpha} A_{\alpha}^{2 \omega} .
$$

Proof. Assume by contradiction that

$$
\frac{\int_{M} u_{\alpha}^{r} \eta_{\alpha}^{r} r_{\alpha}^{2} \mathrm{~d} v_{g}}{\sqrt{\alpha} A_{\alpha}^{2 \omega}} \rightarrow+\infty
$$

Multiplying (2.1) by

$$
\frac{u_{\alpha} \eta_{\alpha}^{r} r_{\alpha}^{2}}{\int_{M} u_{\alpha}^{r} \eta_{\alpha}^{r} r_{\alpha}^{2} \mathrm{~d} v_{g}}
$$

and integrating over $M$, we obtain

$$
\frac{2 A_{\alpha} \int_{M}\left(\Delta_{g} u_{\alpha}\right) u_{\alpha} \eta_{\alpha}^{r} r_{\alpha}^{2} \mathrm{~d} v_{g}}{\int_{M} u_{\alpha}^{r} \eta_{\alpha}^{r} r_{\alpha}^{2} \mathrm{~d} v_{g}}+\frac{2 \alpha A_{\alpha} \int_{M} u_{\alpha}^{2} \eta_{\alpha}^{r} r_{\alpha}^{2} \mathrm{~d} v_{g}}{\int_{M} u_{\alpha}^{r} \eta_{\alpha}^{r} r_{\alpha}^{2} \mathrm{~d} v_{g}}+\frac{2(1-\theta)}{\theta} B_{\alpha} \frac{\int_{M} u_{\alpha}^{s} \eta_{\alpha}^{r} r_{\alpha}^{2} \mathrm{~d} v_{g}}{\int_{M} u_{\alpha}^{r} \eta_{\alpha}^{r} r_{\alpha}^{2} \mathrm{~d} v_{g}}=k_{\alpha}
$$

An integration by parts and Lemma 2.6 lead to

$$
\begin{aligned}
\left|\int_{M}\left(\Delta_{g} u_{\alpha}\right) u_{\alpha} \eta_{\alpha}^{r} r_{\alpha}^{2} \mathrm{~d} v_{g}\right| & \leqslant\left. C\left|\int_{M} \eta_{\alpha}^{r} r_{\alpha}^{2}\right| \nabla u_{\alpha}\right|_{g} \mathrm{~d} v_{g}+\int_{M} u_{\alpha}^{2}\left|\nabla \eta_{\alpha}^{r / 2} r_{\alpha}\right|_{g} \mathrm{~d} v_{g} \mid \\
& \leqslant C\left\|u_{\alpha}\right\|_{\infty}^{2-r} .
\end{aligned}
$$

Hence, by (2.23),

$$
\frac{2 A_{\alpha} \int_{M}\left(\Delta_{g} u_{\alpha}\right) u_{\alpha} \eta_{\alpha}^{r} r_{\alpha}^{2} \mathrm{~d} v_{g}}{\int_{M} u_{\alpha}^{r} \eta_{\alpha}^{r} r_{\alpha}^{2} \mathrm{~d} v_{g}} \leqslant \frac{C A_{\alpha}^{1-2 \omega}\left\|u_{\alpha}\right\|_{\infty}^{2-r}}{\sqrt{\alpha}} \leqslant \frac{C}{\sqrt{\alpha}} \rightarrow 0 .
$$

Since

$$
\frac{2 \alpha A_{\alpha} \int_{M} u_{\alpha}^{2} \eta_{\alpha}^{r} r_{\alpha}^{2} \mathrm{~d} v_{g}}{\int_{M} u_{\alpha}^{r} \eta_{\alpha}^{r} r_{\alpha}^{2} \mathrm{~d} v_{g}} \geqslant 0
$$

we have, by (2.24),

$$
B_{\alpha} \frac{\int_{M} u_{\alpha}^{s} \eta_{\alpha}^{r} r_{\alpha}^{2} \mathrm{~d} v_{g}}{\int_{M} u_{\alpha}^{r} \eta_{\alpha}^{r} r_{\alpha}^{2} \mathrm{~d} v_{g}} \leqslant C
$$

Therefore, by (2.3),

$$
\frac{\int_{M} u_{\alpha}^{s} \eta_{\alpha}^{r} r_{\alpha}^{2} \mathrm{~d} v_{g}}{A_{\alpha}^{s \theta / 2(1-\theta)} \int_{M} u_{\alpha}^{r} \eta_{\alpha}^{r} r_{\alpha}^{2} \mathrm{~d} v_{g}} \leqslant C
$$


Moreover, Lemma 2.4 gives

$$
\int_{M} u_{\alpha}^{s} \eta_{\alpha}^{s} r_{\alpha}^{2} \mathrm{~d} v_{g}-\int_{M} u_{\alpha}^{s} \eta_{\alpha}^{r} r_{\alpha}^{2} \mathrm{~d} v_{g} \leqslant C \int_{M-B_{x_{\alpha}}(c)} u_{\alpha}^{s} \mathrm{~d} v_{g} \leqslant C A_{\alpha}^{2 \omega+(s \theta / 2(1-\theta))} .
$$

It follows from (2.23) and (2.25) that

$$
\frac{\int_{M} u_{\alpha}^{s} \eta_{\alpha}^{s} r_{\alpha}^{2} \mathrm{~d} v_{g}}{A_{\alpha}^{s \theta / 2(1-\theta)} \int_{M} u_{\alpha}^{r} \eta_{\alpha}^{r} r_{\alpha}^{2} \mathrm{~d} v_{g}} \leqslant C
$$

Now let us prove

$$
\int_{M} u_{\alpha}^{s} \eta_{\alpha}^{s} r_{\alpha}^{s} \mathrm{~d} v_{g} \leqslant C \frac{\int_{M} u_{\alpha}^{s} \eta_{\alpha}^{s} r_{\alpha}^{2} \mathrm{~d} v_{g}}{\left(A_{\alpha}^{\omega} \alpha^{1 / 4}\right)^{2-s}}
$$

One has, by Lemma 2.3,

$$
\begin{aligned}
\int_{M} u_{\alpha}^{s} \eta_{\alpha}^{s} r_{\alpha}^{s} \mathrm{~d} v_{g} & =\int_{B_{x_{\alpha}}\left(A_{\alpha}^{\omega} \alpha^{1 / 4}\right)} u_{\alpha}^{s} \eta_{\alpha}^{s} r_{\alpha}^{s} \mathrm{~d} v_{g}+\int_{M-B_{x_{\alpha}}\left(A_{\alpha}^{\omega} \alpha^{1 / 4}\right)} u_{\alpha}^{s} \eta_{\alpha}^{s} r_{\alpha}^{s} \mathrm{~d} v_{g} \\
& \leqslant \int_{B_{x_{\alpha}}\left(A_{\alpha}^{\omega} \alpha^{1 / 4}\right)} u_{\alpha}^{s} \eta_{\alpha}^{s} r_{\alpha}^{s} \mathrm{~d} v_{g}+\frac{C}{\left(A_{\alpha}^{\omega} \alpha^{1 / 4}\right)^{2-s}} \int_{M-B_{x_{\alpha}}\left(A_{\alpha}^{\omega} \alpha^{1 / 4}\right)} u_{\alpha}^{s} \eta_{\alpha}^{s} r_{\alpha}^{2} \mathrm{~d} v_{g}
\end{aligned}
$$

Clearly,

$$
\int_{B_{x_{\alpha}}\left(A_{\alpha}^{\omega} \alpha^{1 / 4}\right)} u_{\alpha}^{s} \eta_{\alpha}^{s} r_{\alpha}^{s} \mathrm{~d} v_{g} \leqslant C A_{\alpha}^{s \theta / 2(1-\theta)}\left(A_{\alpha}^{\omega} \alpha^{1 / 4}\right)^{s} .
$$

Assume by contradiction that

$$
A_{\alpha}^{s \theta / 2(1-\theta)}\left(A_{\alpha}^{\omega} \alpha^{1 / 4}\right)^{s} \geqslant \frac{t_{\alpha}}{\left(A_{\alpha}^{\omega} \alpha^{1 / 4}\right)^{2-s}} \int_{M-B_{x_{\alpha}}\left(A_{\alpha}^{\omega} \alpha^{1 / 4}\right)} u_{\alpha}^{s} \eta_{\alpha}^{s} r_{\alpha}^{2} \mathrm{~d} v_{g},
$$

where $t_{\alpha} \rightarrow+\infty$. We obtain from Lemma 2.3 that

$$
\begin{aligned}
\int_{M-B_{x_{\alpha}}\left(A_{\alpha}^{\omega} \alpha^{1 / 4}\right)} u_{\alpha}^{r} \eta_{\alpha}^{r} r_{\alpha}^{2} \mathrm{~d} v_{g} & \leqslant \int_{M-B_{x_{\alpha}}\left(A_{\alpha}^{\omega} \alpha^{1 / 4}\right)} u_{\alpha}^{r} \eta_{\alpha}^{s} r_{\alpha}^{2} \mathrm{~d} v_{g} \\
& \leqslant \int_{M-B_{x_{\alpha}}\left(A_{\alpha}^{\omega} \alpha^{1 / 4}\right)} u_{\alpha}^{s} \eta_{\alpha}^{s} r_{\alpha}^{2-(r-s)(n / r)} \mathrm{d} v_{g} \\
& \leqslant C\left(A_{\alpha}^{\omega} \alpha^{1 / 4}\right)^{-(r-s)(n / r)} \frac{A_{\alpha}^{s \theta / 2(1-\theta)}\left(A_{\alpha}^{\omega} \alpha^{1 / 4}\right)^{2}}{t_{\alpha}} \\
& \leqslant C \sqrt{\alpha} A_{\alpha}^{2 \omega} .
\end{aligned}
$$

Moreover, we can easily check that

$$
\int_{B_{x_{\alpha}}\left(A_{\alpha}^{\omega} \alpha^{1 / 4}\right)} u_{\alpha}^{r} \eta_{\alpha}^{r} r_{\alpha}^{2} \mathrm{~d} v_{g} \leqslant C \sqrt{\alpha} A_{\alpha}^{2 \omega},
$$


which contradicts (2.23). Hence (2.28) is false and we have proved that

$$
\int_{M-B_{x_{\alpha}}\left(A_{\alpha}^{\omega} \alpha^{1 / 4}\right)} u_{\alpha}^{s} \eta_{\alpha}^{s} r_{\alpha}^{s} \mathrm{~d} v_{g} \leqslant \frac{C}{\left(A_{\alpha}^{\omega} \alpha^{1 / 4}\right)^{2-s}} \int_{M-B_{x_{\alpha}}\left(A_{\alpha}^{\omega} \alpha^{1 / 4}\right)} u_{\alpha}^{s} \eta_{\alpha}^{s} r_{\alpha}^{2} \mathrm{~d} v_{g} .
$$

Inequality (2.27) follows.

From (1.3) with $u=u_{\alpha} r_{\alpha} \eta_{\alpha}$, we obtain

$$
\begin{gathered}
1 \leqslant A \frac{\int_{M}\left|\nabla u_{\alpha} \eta_{\alpha} r_{\alpha}\right|_{g}^{2} \mathrm{~d} v_{g}\left(\int_{M} u_{\alpha}^{s} \eta_{\alpha}^{s} r_{\alpha}^{s} \mathrm{~d} v_{g}\right)^{2(1-\theta) / s \theta}}{\left(\int_{M} u_{\alpha}^{r} \eta_{\alpha}^{r} r_{\alpha}^{r} \mathrm{~d} v_{g}\right)^{2 / r \theta}} \\
+B \frac{\int_{M} u_{\alpha}^{2} \eta_{\alpha}^{2} r_{\alpha}^{2} \mathrm{~d} v_{g}\left(\int_{M} u_{\alpha}^{s} \eta_{\alpha}^{s} r_{\alpha}^{s} \mathrm{~d} v_{g}\right)^{2(1-\theta) / s \theta}}{\left(\int_{M} u_{\alpha}^{r} \eta_{\alpha}^{r} r_{\alpha}^{r} \mathrm{~d} v_{g}\right)^{2 / r \theta}} .
\end{gathered}
$$

Let us prove that

$$
\lim \frac{\int_{M} u_{\alpha}^{2} \eta_{\alpha}^{2} r_{\alpha}^{2} \mathrm{~d} v_{g}\left(\int_{M} u_{\alpha}^{s} \eta_{\alpha}^{s} r_{\alpha}^{s} \mathrm{~d} v_{g}\right)^{2(1-\theta) / s \theta}}{\left(\int_{M} u_{\alpha}^{r} \eta_{\alpha}^{r} r_{\alpha}^{r} \mathrm{~d} v_{g}\right)^{2 / r \theta}}=0
$$

One has, by Hölder's inequality,

$$
\begin{aligned}
& \frac{\int_{M} u_{\alpha}^{2} \eta_{\alpha}^{2} r_{\alpha}^{2} \mathrm{~d} v_{g}\left(\int_{M} u_{\alpha}^{s} \eta_{\alpha}^{s} r_{\alpha}^{s} \mathrm{~d} v_{g}\right)^{2(1-\theta) / s \theta}}{\left(\int_{M} u_{\alpha}^{r} \eta_{\alpha}^{r} r_{\alpha}^{r} \mathrm{~d} v_{g}\right)^{2 / r \theta}} \\
& \quad \leqslant \frac{\left(\int_{M} u_{\alpha}^{s} \eta_{\alpha}^{s} r_{\alpha}^{s} \mathrm{~d} v_{g}\right)^{2(1-\theta) / s \theta}}{\left(\int_{M} u_{\alpha}^{r} \eta_{\alpha}^{r} r_{\alpha}^{r} \mathrm{~d} v_{g}\right)^{(2 / r \theta)-(2 / r)}} \\
& \quad \leqslant \frac{\left(\int_{M} u_{\alpha}^{s} \eta_{\alpha}^{s} r_{\alpha}^{s} \mathrm{~d} v_{g}\right)^{2(1-\theta) / s \theta}}{\left(\int_{M} u_{\alpha}^{r} \eta_{\alpha}^{r} r_{\alpha}^{2} \mathrm{~d} v_{g}\right)^{(1-\theta) / \theta}} \\
& \quad \leqslant\left(\frac{B_{\alpha} \int_{M} u_{\alpha}^{s} \eta_{\alpha}^{s} r_{\alpha}^{s} \mathrm{~d} v_{g}}{\int_{M} u_{\alpha}^{r} \eta_{\alpha}^{r} r_{\alpha}^{2} \mathrm{~d} v_{g}}\right)^{2(1-\theta) / s \theta} \frac{\left(\int_{M} u_{\alpha}^{r} \eta_{\alpha}^{r} r_{\alpha}^{2} \mathrm{~d} v_{g}\right)^{(2(1-\theta) / s \theta)-((1-\theta) / \theta)}}{B_{\alpha}^{2(1-\theta) / s \theta}}
\end{aligned}
$$

Equations (2.27), (2.3) and (2.9) then lead to

$$
\begin{aligned}
& \frac{\int_{M} u_{\alpha}^{2} \eta_{\alpha}^{2} r_{\alpha}^{2} \mathrm{~d} v_{g}\left(\int_{M} u_{\alpha}^{s} \eta_{\alpha}^{s} r_{\alpha}^{s} \mathrm{~d} v_{g}\right)^{2(1-\theta) / s \theta}}{\left(\int_{M} u_{\alpha}^{r} \eta_{\alpha}^{r} r_{\alpha}^{r} \mathrm{~d} v_{g}\right)^{2 / r \theta}} \\
& \leqslant\left(\frac{\int_{M} u_{\alpha}^{s} \eta_{\alpha}^{s} r_{\alpha}^{2} \mathrm{~d} v_{g}}{A_{\alpha}^{s \theta / 2(1-\theta)} \int_{M} u_{\alpha}^{r} \eta_{\alpha}^{r} r_{\alpha}^{2} \mathrm{~d} v_{g}}\right)^{2(1-\theta) / s \theta} \\
& \quad \times \frac{C A_{\alpha}}{\left(A_{\alpha}^{\omega} \alpha^{1 / 4}\right)^{(2-s)(2(1-\theta) / s \theta)}}\left(\int_{M} u_{\alpha}^{r} \eta_{\alpha}^{r} r_{\alpha}^{2} \mathrm{~d} v_{g}\right)^{(2(1-\theta) / s \theta)-((1-\theta) / \theta)} .
\end{aligned}
$$

Therefore, we have, by (2.26),

$$
\frac{\int_{M} u_{\alpha}^{2} \eta_{\alpha}^{2} r_{\alpha}^{2} \mathrm{~d} v_{g}\left(\int_{M} u_{\alpha}^{s} \eta_{\alpha}^{s} r_{\alpha}^{s} \mathrm{~d} v_{g}\right)^{2(1-\theta) / s \theta}}{\left(\int_{M} u_{\alpha}^{r} \eta_{\alpha}^{r} r_{\alpha}^{r} \mathrm{~d} v_{g}\right)^{2 / r \theta}} \leqslant \frac{A_{\alpha}^{1-(r(2-s) / n(r-s))}}{\alpha^{(2-s)(1-\theta) / 2 s \theta}} .
$$


Since

$$
\begin{aligned}
1-\frac{r(2-s)}{n(r-s)} & =\frac{1}{n(r-s)}(n(r-s)-r(2-s)) \\
& =\frac{1}{n(r-s)}((n-2)(r-s)+s(r-2)) \\
& \geqslant 0
\end{aligned}
$$

(2.29) follows.

Now, let us prove that

$$
\frac{\int_{M}\left|\nabla u_{\alpha} \eta_{\alpha} r_{\alpha}\right|_{g}^{2} \mathrm{~d} v_{g}\left(\int_{M} u_{\alpha}^{s} \eta_{\alpha}^{s} r_{\alpha}^{s} \mathrm{~d} v_{g}\right)^{2(1-\theta) / s \theta}}{\left(\int_{M} u_{\alpha}^{r} \eta_{\alpha}^{r} r_{\alpha}^{r} \mathrm{~d} v_{g}\right)^{2 / r \theta}} \rightarrow 0 .
$$

Using (2.27) and (2.26) successively, we obtain

$$
\begin{aligned}
& \frac{\int_{M}\left|\nabla u_{\alpha} \eta_{\alpha} r_{\alpha}\right|_{g}^{2} \mathrm{~d} v_{g}\left(\int_{M} u_{\alpha}^{s} \eta_{\alpha}^{s} r_{\alpha}^{s} \mathrm{~d} v_{g}\right)^{2(1-\theta) / s \theta}}{\left(\int_{M} u_{\alpha}^{r} \eta_{\alpha}^{r} r_{\alpha}^{r} \mathrm{~d} v_{g}\right)^{2 / r \theta}} \\
& \leqslant \frac{C}{\left(A_{\alpha}^{\omega} \alpha^{1 / 4}\right)^{(2-s)(2(1-\theta) / s \theta)}} \frac{\int_{M}\left|\nabla u_{\alpha} \eta_{\alpha} r_{\alpha}\right|_{g}^{2} \mathrm{~d} v_{g}\left(\int_{M} u_{\alpha}^{s} \eta_{\alpha}^{s} r_{\alpha}^{2} \mathrm{~d} v_{g}\right)^{2(1-\theta) / s \theta}}{\left(\int_{M} u_{\alpha}^{r} \eta_{\alpha}^{r} r_{\alpha}^{2} \mathrm{~d} v_{g}\right)^{1 / \theta}} \\
& \leqslant \frac{C A_{\alpha}}{\left(A_{\alpha}^{\omega} \alpha^{1 / 4}\right)^{(2-s)(2(1-\theta) / s \theta)}}\left(\frac{\int_{M} u_{\alpha}^{s} \eta_{\alpha}^{s} r_{\alpha}^{2} \mathrm{~d} v_{g}}{A_{\alpha}^{s \theta / 2(1-\theta)} \int_{M} u_{\alpha}^{r} \eta_{\alpha}^{r} r_{\alpha}^{2} \mathrm{~d} v_{g}}\right)^{2(1-\theta) / s \theta} \\
& \times \frac{\int_{M}\left|\nabla u_{\alpha} \eta_{\alpha} r_{\alpha}\right|_{g}^{2} \mathrm{~d} v_{g}}{\left(\int_{M} u_{\alpha}^{r} \eta_{\alpha}^{r} r_{\alpha}^{2} \mathrm{~d} v_{g}\right)^{(1 / \theta)-(2(1-\theta) / s \theta)}} \\
& \leqslant C \frac{A_{\alpha}^{1-(r(2-s) / n(r-s))}}{\alpha^{(2-s)(1-\theta) / 2 s \theta}} \frac{\int_{M}\left|\nabla u_{\alpha} \eta_{\alpha} r_{\alpha}\right|_{g}^{2} \mathrm{~d} v_{g}}{\left(\int_{M} u_{\alpha}^{r} \eta_{\alpha}^{r} r_{\alpha}^{2} \mathrm{~d} v_{g}\right)^{(1 / \theta)-(2(1-\theta) / s \theta)}} \\
& \leqslant \frac{C A_{\alpha}^{1-(r(2-s) / n(r-s))-2 \omega((1 / \theta)-(2(1-\theta) / s \theta))}}{\alpha^{(2-s)(1-\theta) / 2 s \theta}}\left(\frac{A_{\alpha}^{2 \omega}}{\int_{M} u_{\alpha}^{r} \eta_{\alpha}^{r} r_{\alpha}^{2} \mathrm{~d} v_{g}}\right)^{(1 / \theta)-(2(1-\theta) / s \theta)} \\
& \times \int_{M}\left|\nabla u_{\alpha} \eta_{\alpha} r_{\alpha}\right|_{g}^{2} \mathrm{~d} v_{g} \text {. }
\end{aligned}
$$

Hölder's inequality leads to

$$
\begin{aligned}
\int_{M}\left|\nabla u_{\alpha} \eta_{\alpha} r_{\alpha}\right|_{g}^{2} \mathrm{~d} v_{g}= & \int_{M} \mid \nabla \\
& \left.u_{\alpha}\right|_{g} ^{2} \eta_{\alpha}^{2} r_{\alpha}^{2} \mathrm{~d} v_{g}+2 \int_{M} u_{\alpha} \eta_{\alpha} r_{\alpha}\left\langle\nabla u_{\alpha}, \nabla \eta_{\alpha} r_{\alpha}\right\rangle_{g} \mathrm{~d} v_{g} \\
& +\int_{M} u_{\alpha}^{2}\left|\nabla \eta_{\alpha} r_{\alpha}\right|_{g}^{2} \mathrm{~d} v_{g} \\
\leqslant & \int_{M}\left|\nabla u_{\alpha}\right|_{g}^{2} \eta_{\alpha}^{2} r_{\alpha}^{2} \mathrm{~d} v_{g}+\int_{M} u_{\alpha}^{2}\left|\nabla \eta_{\alpha} r_{\alpha}\right|_{g}^{2} \mathrm{~d} v_{g} \\
& +2\left(\int_{M}\left|\nabla u_{\alpha}\right|_{g}^{2} \eta_{\alpha}^{2} r_{\alpha}^{2} \mathrm{~d} v_{g}\right)^{1 / 2}\left(\int_{M} u_{\alpha}^{2}\left|\nabla \eta_{\alpha} r_{\alpha}\right|_{g}^{2} \mathrm{~d} v_{g}\right)^{1 / 2} .
\end{aligned}
$$


Hence, we have, by Lemma 2.6,

$$
\int_{M}\left|\nabla u_{\alpha} \eta_{\alpha} r_{\alpha}\right|_{g}^{2} \mathrm{~d} v_{g} \leqslant C\left\|u_{\alpha}\right\|_{\infty}^{2-r} .
$$

Finally, noting that

$$
\frac{1}{\theta}-\frac{2(1-\theta)}{s \theta}>\frac{2}{r \theta}-\frac{2(1-\theta)}{s \theta}=1-\frac{2}{n}>0,
$$

we obtain from (2.23) that

$$
\begin{aligned}
& \frac{\int_{M}\left|\nabla u_{\alpha} \eta_{\alpha} r_{\alpha}\right|_{g}^{2} \mathrm{~d} v_{g}\left(\int_{M} u_{\alpha}^{s} \eta_{\alpha}^{s} r_{\alpha}^{s} \mathrm{~d} v_{g}\right)^{2(1-\theta) / s \theta}}{\left(\int_{M} u_{\alpha}^{r} \eta_{\alpha}^{r} r_{\alpha}^{r} \mathrm{~d} v_{g}\right)^{2 / r \theta}} \\
& \leqslant C \frac{A_{\alpha}^{1-(r(2-s) / n(r-s))-2 \omega((1 / \theta)-(2(1-\theta) / s \theta))+2 \omega-1}}{\alpha^{(2-s)(1-\theta) / 2 s \theta}} .
\end{aligned}
$$

However,

$$
\begin{aligned}
1-\frac{r(2-s)}{n(r-s)} & -2 \omega\left(\frac{1}{\theta}-\frac{2(1-\theta)}{s \theta}\right)+2 \omega-1 \\
& =1-\frac{r(2-s)}{n(r-s)}-\frac{r s}{n(r-s)(1-\theta)}+\frac{2 r}{n(r-s)}+\frac{r s \theta}{n(r-s)(1-\theta)}-1 \\
& =\frac{r s}{n(r-s)}\left(1-\frac{1}{1-\theta}+\frac{\theta}{1-\theta}\right) .
\end{aligned}
$$

Relation (2.30) follows. Equations (2.29) and (2.30) contradict (1.3) with $u=u_{\alpha} r_{\alpha} \eta_{\alpha}$. As a consequence, (2.23) is false and Lemma 2.7 is proved.

The last two estimates are important in the third step.

Lemma 2.8. There exists $C>0$ independent of $\alpha$ such that

$$
\frac{1-\left(\int_{M} u_{\alpha}^{r} \eta_{\alpha}^{r} \mathrm{~d} v_{g}\right)^{2 / r \theta}}{\sqrt{\alpha} A_{\alpha}^{2 \omega}} \leqslant C .
$$

Proof. Let $\xi$ be the Euclidean metric on $M$. One then has

$$
\begin{gathered}
\left|\nabla u_{\alpha} \eta_{\alpha}\right|_{\xi}^{2} \leqslant\left|\nabla u_{\alpha} \eta_{\alpha}\right|_{g}^{2}\left(1+C r_{\alpha}^{2}\right) \\
\left(1-C r_{\alpha}^{2}\right) \mathrm{d} v_{\xi} \leqslant \mathrm{d} v_{g} \leqslant\left(1+C r_{\alpha}^{2}\right) \mathrm{d} v_{\xi}, \\
\int_{M}\left|\nabla u_{\alpha} \eta_{\alpha}\right|_{\xi}^{2} \mathrm{~d} v_{\xi} \leqslant \int_{M}\left|\nabla u_{\alpha} \eta_{\alpha}\right|_{g}^{2}\left(1+C r_{\alpha}^{2}\right) \mathrm{d} v_{g} .
\end{gathered}
$$

Hence, we obtain

$$
\begin{aligned}
1-\left(\int_{M} u_{\alpha}^{r} \eta_{\alpha}^{r} \mathrm{~d} v_{\xi}\right)^{2 / r \theta} & \leqslant C\left(1-\int_{M} u_{\alpha}^{r} \eta_{\alpha}^{r} \mathrm{~d} v_{\xi}\right) \\
& \leqslant C\left(\int_{M} u_{\alpha}^{r} \mathrm{~d} v_{g}-\int_{M} u_{\alpha}^{r} \eta_{\alpha}^{r} \mathrm{~d} v_{g}+C \int_{M} u_{\alpha}^{r} \eta_{\alpha}^{r} r_{\alpha}^{2} \mathrm{~d} v_{g}\right) \\
& \leqslant C\left(\int_{M} u_{\alpha}^{r}\left(1-\eta_{\alpha}^{r}\right) \mathrm{d} v_{g}+C \int_{M} u_{\alpha}^{r} \eta_{\alpha}^{r} r_{\alpha}^{2} \mathrm{~d} v_{g}\right) .
\end{aligned}
$$


One easily checks that, if $r>2,2 \omega<r /(r-2)$. Therefore, Lemmas 2.6 and 2.7 lead to Lemma 2.8.

The final lemma that we need is as follows.

Lemma 2.9. There exists $C>0$ independent of $\alpha$ such that

$$
\left(\int_{M} u_{\alpha}^{s} \eta_{\alpha}^{s} \mathrm{~d} v_{\xi}\right)^{2(1-\theta) / s \theta} \leqslant\left(\int_{M} u_{\alpha}^{s} \eta_{\alpha}^{s} \mathrm{~d} v_{g}\right)^{2(1-\theta) / s \theta}+C A_{\alpha}^{1+2 \omega} \sqrt{\alpha} .
$$

Proof. Multiplying (2.1) by $u_{\alpha} r_{\alpha}^{2} \eta_{\alpha}^{r} / A_{\alpha}^{2 \omega} \sqrt{\alpha}$ and integrating over $M$, we obtain

$$
\begin{aligned}
\frac{2 A_{\alpha}^{1-2 \omega}}{\sqrt{\alpha}} \int_{M}\left(\Delta_{g} u_{\alpha}\right) & u_{\alpha} r_{\alpha}^{2} \eta_{\alpha}^{r} \mathrm{~d} v_{g}+2 \sqrt{\alpha} A_{\alpha}^{1-2 \omega} \int_{M} u_{\alpha}^{2} r_{\alpha}^{2} \eta_{\alpha}^{r} \mathrm{~d} v_{g} \\
+ & \frac{2(1-\theta)}{\theta} \frac{B_{\alpha}}{A_{\alpha}^{2 \omega} \sqrt{\alpha}} \int_{M} u_{\alpha}^{s} r_{\alpha}^{2} \eta_{\alpha}^{r} \mathrm{~d} v_{g}=\frac{k_{\alpha}}{A_{\alpha}^{2 \omega} \sqrt{\alpha}} \int_{M} u_{\alpha}^{r} r_{\alpha}^{2} \eta_{\alpha}^{r} \mathrm{~d} v_{g} .
\end{aligned}
$$

One has already shown in the proof of Lemma 2.6 that

$$
\int_{M}\left(\Delta_{g} u_{\alpha}\right) u_{\alpha} r_{\alpha}^{2} \eta_{\alpha}^{r} \mathrm{~d} v_{g} \leqslant C\left\|u_{\alpha}\right\|_{\infty}^{2-r}
$$

Relation (2.3) and Lemma 2.7 then lead to

$$
\int_{M} u_{\alpha}^{s} r_{\alpha}^{2} \eta_{\alpha}^{r} \mathrm{~d} v_{g} \leqslant C \frac{A_{\alpha}^{2 \omega} \sqrt{\alpha}}{B_{\alpha}} \leqslant C A_{\alpha}^{2 \omega+(s \theta / 2(1-\theta))} \sqrt{\alpha} .
$$

And since this result is also true with $\eta=\eta^{s / r}$,

$$
\int_{M} u_{\alpha}^{s} r_{\alpha}^{2} \eta_{\alpha}^{s} \mathrm{~d} v_{g} \leqslant C A_{\alpha}^{2 \omega+(s \theta / 2(1-\theta))} \sqrt{\alpha} .
$$

Noting that $\mathrm{d} v_{\xi} \leqslant\left(1+C r_{\alpha}^{2}\right) \mathrm{d} v_{g}$, we obtain

$$
\begin{aligned}
\left(\int_{M} u_{\alpha}^{s} \eta_{\alpha}^{s} \mathrm{~d} v_{\xi}\right)^{2(1-\theta) / s \theta} & \leqslant\left(\int_{M} u_{\alpha}^{s} \eta_{\alpha}^{s} \mathrm{~d} v_{g}+C \int_{M} u_{\alpha}^{s} \eta_{\alpha}^{s} r_{\alpha}^{2} \mathrm{~d} v_{g}\right)^{2(1-\theta) / s \theta} \\
& \leqslant\left(\int_{M} u_{\alpha}^{s} \eta_{\alpha}^{s} \mathrm{~d} v_{g}\right)^{2(1-\theta) / s \theta}\left(1+C \frac{\int_{M} u_{\alpha}^{s} \eta_{\alpha}^{s} r_{\alpha}^{2} \mathrm{~d} v_{g}}{\int_{M} u_{\alpha}^{s} \eta_{\alpha}^{s} \mathrm{~d} v_{g}}\right)^{2(1-\theta) / s \theta}
\end{aligned}
$$

Inequality (2.32) implies

$$
\frac{\int_{M} u_{\alpha}^{s} \eta_{\alpha}^{s} r_{\alpha}^{2} \mathrm{~d} v_{g}}{\int_{M} u_{\alpha}^{s} \eta_{\alpha}^{s} \mathrm{~d} v_{g}} \rightarrow 0
$$

Consequently,

$$
\begin{aligned}
\left(\int_{M} u_{\alpha}^{s} \eta_{\alpha}^{s} \mathrm{~d} v_{\xi}\right)^{2(1-\theta) / s \theta} \leqslant\left(\int_{M} u_{\alpha}^{s} \eta_{\alpha}^{s} \mathrm{~d} v_{g}\right)^{2(1-\theta) / s \theta} & \\
& +C\left(\int_{M} u_{\alpha}^{s} \eta_{\alpha}^{s} \mathrm{~d} v_{g}\right)^{(2(1-\theta) / s \theta)-1} \int_{M} u_{\alpha}^{s} \eta_{\alpha}^{s} r_{\alpha}^{2} \mathrm{~d} v_{g} .
\end{aligned}
$$


One deduces from (2.32) and Lemma 2.2 that

$$
\begin{aligned}
& \left(\int_{M} u_{\alpha}^{s} \eta_{\alpha}^{s} \mathrm{~d} v_{\xi}\right)^{2(1-\theta) / s \theta} \\
& \leqslant\left(\int_{M} u_{\alpha}^{s} \eta_{\alpha}^{s} \mathrm{~d} v_{g}\right)^{2(1-\theta) / s \theta} \\
& \quad+C\left(\int_{M} u_{\alpha}^{s} \mathrm{~d} v_{g}\right)^{(2(1-\theta) / s \theta)-1}\left(\frac{\int_{M} u_{\alpha}^{s} \eta_{\alpha}^{s} \mathrm{~d} v_{g}}{\int_{M} u_{\alpha}^{s} \mathrm{~d} v_{g}}\right)^{(2(1-\theta) / s \theta)-1} \int_{M} u_{\alpha}^{s} \eta_{\alpha}^{s} r_{\alpha}^{2} \mathrm{~d} v_{g} \\
& \quad\left(\int_{M} u_{\alpha}^{s} \eta_{\alpha}^{s} \mathrm{~d} v_{g}\right)^{2(1-\theta) / s \theta}+C A_{\alpha}^{1+2 \omega} \sqrt{\alpha} .
\end{aligned}
$$

This ends the proof of the lemma.

Step 3. Conclusion.

One has, by definition of $A_{0}$,

$$
\left(\int_{M} u_{\alpha}^{r} \eta_{\alpha}^{r} \mathrm{~d} v_{\xi}\right)^{2 / r \theta} \leqslant A_{0} \int_{M}\left|\nabla u_{\alpha} \eta_{\alpha}\right|_{\xi}^{2} \mathrm{~d} v_{\xi}\left(\int_{M} u_{\alpha}^{s} \eta_{\alpha}^{s} \mathrm{~d} v_{\xi}\right)^{2(1-\theta) / s \theta}
$$

and, by Lemma 2.6 and (2.31),

$$
\int_{M}\left|\nabla u_{\alpha} \eta_{\alpha}\right|_{\xi}^{2} \mathrm{~d} v_{\xi} \leqslant \int_{M}\left|\nabla u_{\alpha}\right|_{g}^{2} \eta_{\alpha}^{2} \mathrm{~d} v_{g}+C\left\|u_{\alpha}\right\|_{\infty}^{2-r} .
$$

Hence, we obtain from Lemma 2.9 that

$$
\left(\int_{M} u_{\alpha}^{r} \eta_{\alpha}^{r} \mathrm{~d} v_{\xi}\right)^{2 / r \theta} \leqslant A_{0} \int_{M}\left|\nabla u_{\alpha}\right|_{g}^{2} \eta_{\alpha}^{2} \mathrm{~d} v_{g}\left(\int_{M} u_{\alpha}^{s} \eta_{\alpha}^{s} \mathrm{~d} v_{g}\right)^{2(1-\theta) / s \theta}+C A_{\alpha}^{4 \omega} \sqrt{\alpha}
$$

The definition of $u_{\alpha}$ leads to

$$
1=\left(\frac{1}{\mu_{\alpha}} \int_{M}\left|\nabla u_{\alpha}\right|_{g}^{2} \mathrm{~d} v_{g}+\frac{\alpha}{\mu_{\alpha}} \int_{M} u_{\alpha}^{2} \mathrm{~d} v_{g}\right) A_{\alpha} .
$$

Combining (2.33) and (2.34), we obtain

$$
\begin{aligned}
1-\left(\int_{M} u_{\alpha}^{r} \eta_{\alpha}^{r} \mathrm{~d} v_{g}\right)^{2 / r \theta} \geqslant & -A_{0} \int_{M}\left|\nabla u_{\alpha}\right|_{g}^{2} \eta_{\alpha}^{2} \mathrm{~d} v_{g}\left(\int_{M} u_{\alpha}^{s} \eta_{\alpha}^{s} \mathrm{~d} v_{g}\right)^{2(1-\theta) / s \theta} \\
& +\frac{A_{\alpha}}{\mu_{\alpha}} \int_{M}\left|\nabla u_{\alpha}\right|_{g}^{2} \mathrm{~d} v_{g}+\frac{\alpha A_{\alpha}}{\mu_{\alpha}} \int_{M} u_{\alpha}^{2} \mathrm{~d} v_{g}-C A_{\alpha}^{4 \omega} \sqrt{\alpha} .
\end{aligned}
$$

Then, noting that

$$
A_{\alpha} \int_{M} u_{\alpha}^{2} \mathrm{~d} v_{g} \geqslant C A_{\alpha}^{2 \omega}
$$


and dividing by $A_{\alpha}^{2 \omega} \sqrt{\alpha}$, it follows that

$$
\begin{aligned}
\frac{1-\left(\int_{M} u_{\alpha}^{r} \eta_{\alpha}^{r} \mathrm{~d} v_{g}\right)^{2 / r \theta}}{\sqrt{\alpha} A_{\alpha}^{2 \omega}} \geqslant-\frac{A_{0}}{\sqrt{\alpha}} \int_{M}\left|\nabla u_{\alpha}\right|_{g}^{2} \eta_{\alpha}^{2} \mathrm{~d} v_{g} \frac{\left(\int_{M} u_{\alpha}^{s} \eta_{\alpha}^{s} \mathrm{~d} v_{g}\right)^{2(1-\theta) / s \theta}}{A_{\alpha}^{2 \omega}} & \\
& +\frac{A_{\alpha}^{1-2 \omega}}{\mu_{\alpha} \sqrt{\alpha}} \int_{M}\left|\nabla u_{\alpha}\right|_{g}^{2} \mathrm{~d} v_{g}+\frac{\sqrt{\alpha}}{\mu_{\alpha}}-C A_{\alpha}^{2 \omega} .
\end{aligned}
$$

Finally, since

$$
\begin{gathered}
\frac{1}{\mu_{\alpha}} \geqslant A_{0}, \\
\frac{\left(\int_{M} u_{\alpha}^{s} \eta_{\alpha}^{s} \mathrm{~d} v_{g}\right)^{2(1-\theta) / s \theta}}{A_{\alpha}} \leqslant 1,
\end{gathered}
$$

we find

$$
\frac{1-\left(\int_{M} u_{\alpha}^{r} \eta_{\alpha}^{r} \mathrm{~d} v_{g}\right)^{2 / r \theta}}{\sqrt{\alpha} A_{\alpha}^{2 \omega}} \geqslant \frac{A_{0} A_{\alpha}^{1-2 \omega}}{\sqrt{\alpha}} \int_{M}\left|\nabla u_{\alpha}\right|_{g}^{2}\left(1-\eta_{\alpha}^{2}\right) \mathrm{d} v_{g}+A_{0} \sqrt{\alpha}-C A_{\alpha}^{2 \omega} .
$$

By Lemma 2.8, the left member is bounded while the right one converges to $+\infty$. This ends the proof of the theorem.

\section{Some applications}

\subsection{The best-constant problem for the logarithmic Sobolev inequality}

In this subsection we prove Corollary 1.2. Fix $r=2$. One then has the following inequalities:

$$
\left(\int_{M}|u|^{2} \mathrm{~d} v_{g}\right)^{1+(2 / n((2-s) / s))} \leqslant\left(A \int_{M}|\nabla u|_{g}^{2} \mathrm{~d} v_{g}+B \int_{M}|u|^{2} \mathrm{~d} v_{g}\right)\left(\int_{M}|u|^{s} \mathrm{~d} v_{g}\right)^{4 / n(2-s)},
$$

where $1 \leqslant s<2$. Let us denote them by $I_{s}(A, B)$. We proved in $\S 2$ above that all these inequalities hold with their first best constant. Set

$$
\begin{aligned}
& A(s)=\inf \left\{A \in \mathbb{R} \text { s.t. } \exists B \in \mathbb{R} \text { for which } I_{s}(A, B) \text { is valid }\right\}, \\
& B(s)=\inf \left\{B \in \mathbb{R} \text { s.t. } I_{s}(A(s), B) \text { is valid }\right\} .
\end{aligned}
$$

It is clear that $I_{s^{\prime}}(A, B)$ implies $I_{s}(A, B)$ when $s^{\prime}>s$. Therefore, $A(s)$ is increasing. According to $[\mathbf{3}], A(s)$ is bounded by a constant independent of $s$. Hence, $A(s)$ converges to a constant $A(2)$ as $s \rightarrow 2$. If $s^{\prime}>s, I_{s}\left(A\left(s^{\prime}\right), B\left(s^{\prime}\right)\right)$ holds. One can then set

$$
A^{\prime}(s)=\inf \left\{A \in \mathbb{R} \text { s.t. } I_{s}\left(A, B\left(s^{\prime}\right)\right) \text { is valid }\right\} .
$$

Thereafter, by definition of $A^{\prime}(s)$, for all $\epsilon>0$, there exists $u \in C^{\infty}(M)$ such that $\|u\|_{s}=1$ and

$$
A^{\prime}(s) \int_{M}|\nabla u|_{g}^{2} \mathrm{~d} v_{g}+B\left(s^{\prime}\right) \int_{M}|u|^{2} \mathrm{~d} v_{g} \leqslant\left(\int_{M}|u|^{2} \mathrm{~d} v_{g}\right)^{1+(2 /(n(2-s) / s))}+\epsilon .
$$


Adding the previous inequality with $I_{s}(A(s), B(s))(u)$ and noting that $A(s) \leqslant A^{\prime}(s)$, we easily obtain $B\left(s^{\prime}\right)-B(s) \leqslant V_{g}(M)^{(2 / s)-1} \epsilon$. Since $\epsilon$ is arbitrary, we have proved that $B(s)$ is decreasing and converges to a constant $B(2)$ as $s \rightarrow 2$. Now, taking the limit in $I_{s}(A(s), B(s))$ as $s \rightarrow 2$, we obtain that for all $u>0$ such that $\|u\|_{2}=1$ the logarithmic Sobolev inequality

$$
\int_{M} u^{2} \ln u^{2} \mathrm{~d} v_{g} \leqslant \frac{1}{2} n \ln \left(A(2) \int_{M}|\nabla u|_{g}^{2} \mathrm{~d} v_{g}+B(2)\right) .
$$

Clearly, $A(2)=A_{0}(2,2,0, n)=(2 / n \pi \mathrm{e})$ is optimal and the inequality is optimal in the sense that no constant can be lowered. This proves Corollary 1.2 .

\subsection{Heat-kernel upper-bounds estimates}

We discuss here one application of the estimates of the heat-kernel upper bounds. When $M$ is a complete manifold (not necessarily compact), it is well known (see, for example, $[\mathbf{6}]$ ) that all the previous inequalities are equivalent to

$$
\left\|P_{t}\right\|_{1, \infty} \leqslant \frac{C}{t^{n / 2}},
$$

where $\left(P_{t}\right)_{t>0}$ is the heat semigroup on $M$. Moreover, when $M$ is the Euclidean space $\mathbb{R}^{n}$, we have

$$
\left\|P_{t}\right\|_{1, \infty}=\frac{1}{(4 \pi t)^{n / 2}} .
$$

Hence, it is quite obvious that, on a manifold, we should have the small-time estimate

$$
\left\|P_{t}\right\|_{1, \infty} \sim \frac{1}{(4 \pi t)^{n / 2}}
$$

Corollary 1.3 gives additional information on this estimate when $M$ is compact. In order to prove it, we need the following theorem from Bakry (see [2] for a detailed proof in the more general case of the Markov diffusion generators).

Theorem 3.1. Let us assume that, for all $u \in C^{\infty}(M)$ such that $u>0$ and $\|u\|_{2}=1$,

$$
\int_{M} u^{2} \ln u^{2} \mathrm{~d} v_{g} \leqslant \phi\left(\int_{M}|\nabla u|_{g}^{2} \mathrm{~d} v_{g}\right),
$$

where $\phi: \mathbb{R}_{+} \rightarrow \mathbb{R}_{+}$is concave, increasing and of class $C^{1}$. One then has for all $1 \leqslant p<$ $q \leqslant \infty$

$$
\left\|P_{t}\right\|_{p, q} \leqslant \mathrm{e}^{m}
$$

where

$$
t=\int_{p}^{q} \phi^{\prime}(v(s)) \frac{\mathrm{d} s}{4(s-1)} \quad \text { and } \quad m=\int_{p}^{q}\left(\phi(v(s))-v(s) \phi^{\prime}(v(s))\right) \frac{\mathrm{d} s}{s^{2}},
$$

provided we find a function $v \geqslant 0$ for which these two integrals are finite. 
Set

$$
v(s)=\frac{\lambda s^{2}}{s-1}-\frac{1}{2} n \pi \mathrm{e} B(2),
$$

where $\lambda \geqslant \frac{1}{8} n \pi \mathrm{e} B(2)$ is a parameter and $B(2)$ is the constant introduced in the previous subsection. One has

$$
\phi(x)=\frac{1}{2} n \ln \left(\frac{2}{n \pi \mathrm{e}} x+B(2)\right) .
$$

It is an easy matter to check that

$$
\phi^{\prime}(v(s))=\frac{1}{2} n \frac{s-1}{\lambda s^{2}}
$$

and that

$$
\phi(v(s))-v(s) \phi^{\prime}(v(s))=\frac{1}{2} n \ln \left(\frac{2 \lambda s^{2}}{n \pi \mathrm{e}^{2}(s-1)}\right)+\frac{n^{2} \pi \mathrm{e} B(2)(s-1)}{4 \lambda s^{2}} .
$$

Some easy computations then lead to

$$
t=\int_{1}^{\infty} \frac{n}{8 \lambda s^{2}} \mathrm{~d} s=\frac{n}{8 \lambda}
$$

and

$$
\begin{aligned}
m & =\frac{1}{2} n \int_{1}^{\infty} \ln \left(\frac{2 \lambda s^{2}}{n \pi \mathrm{e}^{2}(s-1)}\right) \frac{\mathrm{d} s}{s^{2}}+\frac{n^{2} \pi \mathrm{e} B(2)}{4 \lambda} \int_{1}^{\infty} \frac{s-1}{s^{4}} \mathrm{~d} s \\
& =\frac{1}{2} n \ln \left(\frac{2 \lambda}{n \pi \mathrm{e}^{2}}\right)+\frac{1}{2} n \int_{1}^{\infty} \ln \left(\frac{s^{2}}{s-1}\right) \frac{\mathrm{d} s}{s^{2}}+\frac{n^{2} \pi \mathrm{e} B(2)}{24 \lambda} \\
& =\frac{1}{2} n \ln \left(\frac{2 \lambda}{n \pi \mathrm{e}^{2}}\right)+n+\frac{n^{2} \pi \mathrm{e} B(2)}{24 \lambda} \\
& =\frac{1}{2} n \ln \left(\frac{2 \lambda}{n \pi}\right)+\frac{n^{2} \pi \mathrm{e} B(2)}{24 \lambda} .
\end{aligned}
$$

Since $\lambda=n / 8 t$,

$$
m=\frac{1}{2} n \ln \left(\frac{1}{4 \pi t}\right)+\frac{1}{3} n \pi \mathrm{e} B(2) t
$$

It follows that

$$
\left\|P_{t}\right\|_{1, \infty} \leqslant \frac{1}{(4 \pi t)^{n / 2}} \mathrm{e}^{n \pi \mathrm{e} B(2) t / 3}
$$

with $0<t \leqslant(\pi \mathrm{e} B(2))^{-1}$. This yields Corollary 1.3.

\section{References}

1. T. Aubin, Problèmes isopérimétriques et espaces de Sobolev, J. Diff. Geom. 11 (1976), 573-598.

2. D. BAKRY, L'hypercontractivité et son utilisation en théorie des semigroupes, Ecole d'Eté de Probabilités de St-Flour, Lecture Notes in Mathematics, vol. 1581, pp. 1-114 (Springer, 1994). 
3. D. Bakry, T. Coulhon, M. Ledoux And L. SAloff-Coste, Sobolev inequalities in disguise, Indiana J. Math., 44 (1995), 1033-1074.

4. E. CARlen, Superadditivity of Fisher's information and logarithmic Sobolev inequalities, J. Funct. Analysis 101 (1991), 194-211.

5. E. Carlen And M. Loss, Sharp constant in Nash's inequality, Duke Math. J. Int. Math. Res. Not. 7 (1993), 213-215.

6. E. B. DAviEs, Heat kernels and spectral theory (Cambridge University Press, 1989).

7. O. Druet, The best constant problem in Sobolev inequalities, Math. Annln 314 (1999), 327-346.

8. O. Druet, E. Hebey And M. Vaugon, Optimal Nash's inequalities on Riemannian manifolds: the influence of geometry, Int. Math. Res. Not. 14 (1999), 735-779.

9. D. GILBARG AND N. S. TRÜDINGER, Elliptic partial differential equations of second order, Grundlehren der Mathematischen Wissenschaften, vol. 224 (Springer, 1977)

10. E. HeBEy, Nonlinear analysis on manifolds: Sobolev spaces and inequalities, Courant Lecture Notes in Mathematics, vol. 5 (1999).

11. E. Humbert, Best constants in the $L^{2}$-Nash inequality, Proc. R. Soc. Edinb. 131 (2001), 621-646.

12. J. Moser, On Harnack's theorem for elliptic differential equations, Commun. Pure Appl. Math. 14 (1961), 577-591.

13. J. NAsh, Continuity of solutions of parabolic and elliptic equations, Am. J. Math. 80 (1958), 931-954.

14. G. Talenti, Best constants in Sobolev inequality, Annli Mat. Pura Appl. 110 (1976), 353-372. 